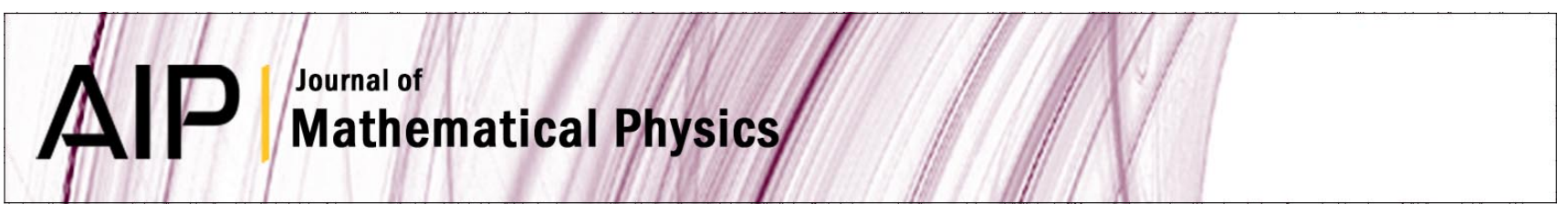

\title{
Uniqueness of Gibbs states in one-dimensional antiferromagnetic model with long-range interaction
}

Azer Kerimov

Citation: J. Math. Phys. 40, 4956 (1999); doi: 10.1063/1.533009

View online: http://dx.doi.org/10.1063/1.533009

View Table of Contents: http://jmp.aip.org/resource/1/JMAPAQ/v40/i10

Published by the American Institute of Physics.

\section{Additional information on J. Math. Phys.}

Journal Homepage: http://jmp.aip.org/

Journal Information: http://jmp.aip.org/about/about_the_journal

Top downloads: http://jmp.aip.org/features/most_downloaded

Information for Authors: http://jmp.aip.org/authors

\section{ADVERTISEMENT}

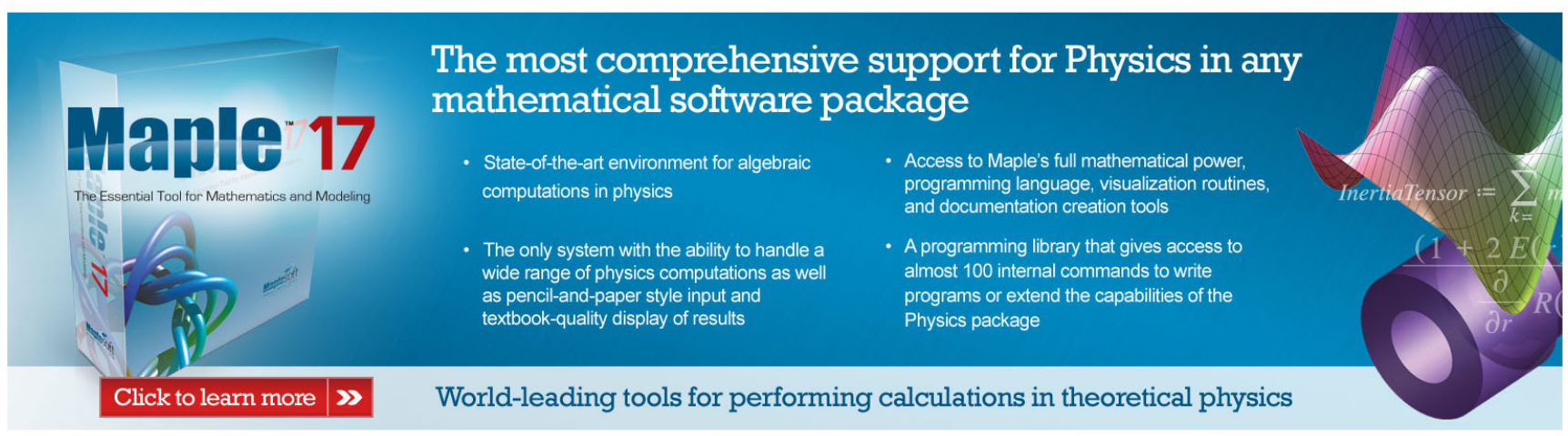




\title{
Uniqueness of Gibbs states in one-dimensional antiferromagnetic model with long-range interaction
}

\author{
Azer Kerimov \\ Department of Mathematics, Bilkent University, 06533 Bilkent, Ankara, Turkey
}

(Received 28 May 1998; accepted for publication 2 April 1999)

Uniqueness of Gibbs states in the one-dimensional antiferromagnetic model with very long-range interaction is established. (c) 1999 American Institute of Physics. [S0022-2488(99)03309-5]

\section{INTRODUCTION}

We study a model on the lattice $\mathbf{Z}^{1}$ with the Hamiltonian

$$
\mathbf{H}(\varphi(x))=\sum_{x, y \in \mathbf{Z}^{1} ; x>y} U(x-y) \varphi(x) \varphi(y)-\mu \sum_{x \in \mathbf{Z}^{1}} \varphi(x),
$$

where the spin variable $\varphi(x)$ takes the values 0 and $1, \mu$ is a chemical potential. The antiferromagnetic potential $U(x)>0$ satisfies the following conditions:

(1) $U(x+y)+U(x-y)>2 U(x) ; x, y \in \mathbf{Z}^{1}, x>y$.

(2) The function $U(x)$ can be extended to a twice continuously differentiable function such that $U(x) \sim A(x)^{-\gamma}, U^{\prime} \sim-A \gamma x^{-\gamma-1}$ and $U^{\prime \prime}(x) \sim A \gamma(\gamma+1) x^{-\gamma-2}$ at $x \rightarrow \infty$; where $\gamma>1$, and $A$ is a strong positive constant.

The first convexity condition plays a significant role for the structure of the set of all ground states of the model (1). The second condition determines the character of the potential's decrease at infinity and is important in further calculations.

The hypothesis on the uniqueness of the Gibbs states in the model (1) was stated by Sinai in 1983 (see Ref. 1, Problem 1).

It is well known that the condition $\Sigma_{x \in \mathbf{Z}^{1}, x>0} x U(x)<\infty$ automatically implies the uniqueness of the Gibbs states. ${ }^{2-4}$ We investigate the phase transition problem in the model (1) in the alternative case, when $U(x) \sim A x^{-\gamma}$, where $\gamma=1+\alpha, 0<\alpha<1$.

The ferromagnetic version of this model [when the potential $U(x)$ is negative] was considered by Dyson in his well-known papers. ${ }^{5,6}$ He proved the existence of two extreme limit Gibbs states $P^{+}$and $P^{-}$corresponding to the ground states $\varphi(x)=+1$ and $\varphi(x)=-1$ at low temperatures.

A series of papers has been devoted to the investigation of the antiferromagnetic model (1). ${ }^{1,7-13}$

The validity of Sinai's hypothesis for rational values of the density (for almost each value of the external field) at low temperatures was proved in Ref. 13.

The main purpose of this paper is to extend the result of Ref. 13 to all values of the external field and to all values of the temperature. $\beta^{-1}$.

Theorem 1: The model (1) has a unique limit Gibbs state at all values of the temperature

Let us introduce necessary definitions. The set of all periodic configurations we denote by $\Phi^{\mathrm{per}}$. For every $\varphi \in \Phi^{\mathrm{per}}$, we define $q=\sum_{y=x+1}^{x+p} \varphi(x)$, where $p$ is the period of $\varphi$. It is obvious that $q$ does not depend on $x$. Therefore, the density of each periodic configuration is $\kappa=q / p$. It is more convenient to work with the reciprocal of the density, $\eta(\varphi(x))=p / q$, which represents the average distance between neighboring points at which $\varphi(x)=1$. For every configuration $\varphi \in \Phi^{\text {per }}$ the mean energy $h(\varphi)$ is defined as follows: 


$$
h(\varphi(x))=\frac{1}{p} \sum_{y=x+1}^{x+p} \varphi(x) \sum_{z>0} U(z) \varphi(y+z) .
$$

The last expression does not depend on $x$.

The following definition is useful for describing the zero temperature phase diagram of the model (1).

We fix a positive rational number $p / q$.

A configuration $\varphi_{0}(x) \in \Phi^{\text {per }}$ with $\eta\left(\varphi_{0}(x)\right)=p / q$ is called a special ground state ${ }^{1}$ if

$$
h(\varphi(x))=\inf _{\varphi \in \Phi^{\mathrm{per}}, \eta(\varphi)=p / q} h(\varphi) .
$$

Hubbard's criterion (Refs. 1 and 7): Let $\varphi \in \Phi^{\mathrm{per}}$ and $r_{i}(x ; \varphi)$ denotes the distance between a particle placed at $x \in \mathbf{Z}^{1}$ and $i$ th particle on the right. If for each $x$ and $i$

$$
[i \eta] \leqslant r_{i}(x ; \varphi) \leqslant[i \eta]+1,
$$

(the square brackets denote the integer part of the enclosed number) then $\varphi$ is a special ground state.

The existence of configurations satisfying Hubbard's criterion (the special ground states) is shown in Ref. 1. The remarkable elegant formula for the special ground states was offered by Aubry. Here we give the construction of the special ground states for each fixed rational value of the density $\kappa{ }^{1}$

Every rational number $p / q$ has a unique decomposition into a finite continued fraction:

$p / q=\left[n_{0}, n_{1}, \ldots, n_{s}\right]$, this means that

$$
n_{0}+\frac{1}{n_{1}+\frac{1}{n_{2}+\ldots+\frac{1}{n_{s}}}} .
$$

The ground state for a configuration with $\eta=\left[n_{0}, n_{1}, \ldots, n_{s}\right]$ will be constructed by recursion.

(1) $\eta=n_{0} \geqslant 1, n_{0}$ is an integer. The periodic configuration with equally distant $x$ at which $\varphi(x)=1$ satisfies Hubbard's criterion i.e., is a special ground state. In this case $r_{i}(x ; \varphi)=i n_{0}, i$ $>0$.

(2) $\eta=n_{0}+1 / n_{1}$, where $n_{0}$ and $n_{1}$ are integers, $n_{0} \geqslant 1, n_{1}>1$. Then the $\left(n_{0} n_{1}+1\right)$ periodic configuration

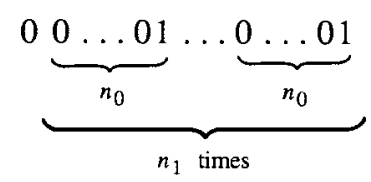

also satisfies Hubbard's criterion and is a special ground state.

(3) $\eta=\left[n_{0}, n_{1}, \ldots, n_{s}\right]$, where $n_{0}, n_{1}, \ldots, n_{s}$ are integers, $n_{0}, n_{1}, \ldots, n_{s} \geqslant 1$. For $s=0$ and $s$ $=1$ the required configurations are already constructed. Suppose we have already constructed a ground state with $s=m$ and $\kappa=\left[n_{0}, n_{1}, \ldots, n_{m}\right]$. Then the following configuration with $s=m$ +1 and $\kappa=\left[n_{0}, n_{1}, \ldots, n_{m+1}\right]$ is constructed as

$$
\varphi\left(n_{0}, \ldots, n_{m+1}\right)=\varphi\left(n_{0}, \ldots, n_{m-1}\right) \underbrace{\varphi\left(n_{0}, \ldots n_{m}\right) \ldots \varphi\left(n_{0}, \ldots, n_{m}\right)}_{n_{m+1} \text { times }} .
$$

Here, $\varphi\left(n_{0}, \ldots, n j\right), j=m-1, m, m+1$, are the blocks from which the ground states for $\eta$ $=\left[n_{0}, \ldots, n_{j}\right]$ are obtained by periodic continuations.

The constructed configuration satisfies Hubbard's criterion and therefore is a special ground state for $\eta=\left[n_{0}, n_{1}, \ldots, n_{m}, n_{m+1}\right] .^{1}$ 
The following explicit expression for the mean energy of the special ground state follows from Hubbard's criterion: ${ }^{1}$

$$
h_{\kappa}=\kappa \sum_{i=1}^{\infty} U\left(m_{i}\right) \pi_{i}+U\left(m_{i}+1\right)\left(1-\pi_{i}\right)
$$

where $m_{i}=[i \eta], \pi_{i}=1+m_{i}-i \eta$.

This formula shows that the function of mean energy as a function of the density $\kappa$ is continuous on the set of all rationals and can be extended to a continuous function defined on whole segment $[0,1]$.

Theorem 2: (Refs. 9 and 1.) (1) The function $h_{\kappa}$ is convex.

(2) In each rational point the function $h_{\kappa}$ has a left-hand derivative $\mu_{\kappa}^{-}$and a right-hand derivative $\mu_{\kappa}^{+}$, with $\mu_{\kappa}^{+}>\mu_{\kappa}^{-}$.

(3) The Lebesgue measure of the complement of the set $\cup_{\kappa}\left(\mu_{\kappa}^{-}, \mu_{\kappa}^{+}\right)$in the real line $\mathbf{R}$ is zero.

The following theorem gives the full description of the set of all special ground states of the model (1) at rational densities.

Theorem 3: (Ref. 12.) Suppose that the value of the external field $\mu$ of the model (1) belongs to the interval $\left(\mu_{\kappa}^{-}, \mu_{\kappa}^{+}\right)$for some number $\kappa=q / p$. Then the special ground state of the model (1) is unique up to translations.

Following Theorem 4 generalizes the main result of Ref. 13 for all values of the temperature and is a special case (rational densities) of Theorem 1.

Theorem 4: Suppose that the value of the external field $\mu$ of the model (1) belongs to the interval $\left(\mu_{\kappa}^{-}, \mu_{\kappa}^{+}\right)$for some number $\kappa=q / p$.

Then the model (1) has a unique limit Gibbs state at all values of the temperature $\beta^{-1}$.

Suppose that the value of the external field $\mu$ of the model (1) belongs to the interval $\left(\mu_{\kappa}^{-}, \mu_{\kappa}^{+}\right)$for some number $\kappa=q / p$.

Let us consider an arbitrary configuration $\varphi(x)$. We say that $\varphi([a, b]) ; a, b \in \mathbf{Z}^{1}$ is a preregular phase, if there exists a special ground state $\varphi_{\kappa}$, such that the restriction of this configuration to $[a, b]$ coincides with $\varphi([a, b])$. We say that $\varphi([c, d]) ; c, d \in \mathbf{Z}^{1}$ is a regular phase, if there exists a preregular phase $\varphi([a, b]) ; a, b \in \mathbf{Z}^{1}$, such that $c-a>d_{0} p$ and $b-d>d_{0} p$. Thus, right and left $d_{0} p$ extensions of a regular phase are ground states.

Let us consider a set $A=\cup_{i}\left[a_{i}, b_{i}\right]$, where $\varphi\left(\left[a_{i}, b_{i}\right]\right)$ is a regular phase and supp $P B$ is the complement of $A$ in $\mathbf{Z}^{1}$. The connected components of supp $P B$ defined in such a way are called supports of precontours and are denoted by supp $P K$ : supp $P K=\cup_{i \in \operatorname{Ind}} \operatorname{supp} P K_{i}$.

For each fixed rational density $\kappa$ the constant $d_{0}$ satisfies some technical conditions. ${ }^{13}$ In this work we do not need the explicit value of $d_{0}$.

Definition 1 (Ref. 13): The pair $P K=\left(\operatorname{supp} P K, \varphi^{\prime}(\operatorname{supp} P K)\right)$ is called a precontour. The set of all precontours is called a preboundary $P B$ of the configuration $\varphi^{\prime}(x)$. Two precontours $P K_{1}$ and $P K_{2}$ are said to be connected if $\operatorname{dist}\left(\operatorname{supp} P K_{1}, \operatorname{supp} P K_{2}\right)<N_{b}$. The set of precontours ( $P K_{i} ; i \in$ Ind) is called connected if for any two precontours $P K_{c}$ and $P K_{d} ; c, d \in$ Ind there exists a collection $\left(P K_{j_{1}}=P K_{c}, \ldots, P K_{j_{i}}, \ldots, P K_{j_{n-1}}, P K_{j_{n}}=P K_{d}\right) ; j_{i} \in$ Ind, $i=1, \ldots, n$; such that any two precontours $P K_{j_{i}}$ and $P K_{j_{i+1}}, i=1, \ldots, n-1$ are connected. Let $\cup_{i=1}^{n} P K_{i}$ be some maximal connected component of the preboundary $P B$. Suppose that $\operatorname{supp} P K_{i}=\left[a_{i}, b_{i}\right]$ and $b_{i}<a_{i+1} ; i$ $=, \ldots, n-1$.

The pair $K=\left(\operatorname{supp} K, \varphi^{\prime}(\operatorname{supp} P K)\right)$, where supp $K=\left[a_{1}, b_{n}\right]$ is called a contour. The set of all contours is called a boundary $B$ of the configuration $\varphi^{\prime}(x)$.

In this work we do not need the exact value of the constant $N_{b} .{ }^{12}$ From Ref. 12 it becomes clear that $\lim _{p \rightarrow \infty} N_{b}=\infty$. Thus, for irrational values of the density $\kappa N_{b}$ is not defined, but as will be seen below, we do not need to define $N_{b}$ for irrational densities.

Note that $\operatorname{supp} K=\left(\cup_{i=1}^{n} \operatorname{supp} P K_{i}\right) \cup\left(\left[a_{1}, b_{n}\right]-\left(\cup_{i=1}^{n} \operatorname{supp} P K_{i}\right)\right)=\operatorname{supp}^{1} K \cup \operatorname{supp}^{2} K$. 
The sets $\operatorname{supp}^{1} K$ and $\operatorname{supp}^{2} K$ will be, respectively, called the essential and regular parts of the support supp $K$.

Let the boundary conditions $\bar{\varphi}(x)=[\varphi(x), x \in(-\infty,-V-1] \cup[V+1, \infty)]$ be fixed. The set of all configurations $\varphi(x) ; x \in[-V, V]$ we denote via $\Phi(V)$.

It is obvious that for each contour $K$, such that supp $K \in\left[-V+\left(d_{0}+1\right) p, V-\left(d_{0}+1\right) p\right]$, there exists a configuration $\psi_{K}([-V, V])$ such that the boundary of the configuration $\psi_{K}([-V, V])$ includes the contour $K$ only:

$$
B\left(\psi_{K}([-V, V])\right)=K
$$

Let $\operatorname{supp} K=[a, b]$. It is obvious that the restrictions of the configuration $\psi_{K}([-V, V])$ to the segments $[-V, a-1]$ and $[b+1, V]$ coincide with two ground states $\varphi_{\kappa}^{1}(x)$ and $\varphi_{\kappa}^{2}(x)$.

A contour $K$ is called an interface contour, if $\varphi_{\kappa}^{1}(x) \neq \varphi_{\kappa}^{2}(x)$.

Note that, $\varphi_{\kappa}^{1}(x)$ can be obtained by some shifting of the configuration $\varphi_{\kappa}^{2}(x)$.

An interface contour will be denoted as $I K$.

Let $K$ be a usual contour (not an interface contour) $K$, supp $K \subset[-V, V]$ and $\psi \mathrm{K}(\mathrm{x})$ $=\psi([-V, V])$ if $x \in[-V, V]$, and $\bar{\varphi}(x)$ if $x \in(-\infty,-V-1] \cup[V+1, \infty) ; \quad I K$, supp $I K \subset$ $[-V, V]$ be an interface contour and $\psi_{I K}(x)=\psi([-V, V])$ if $x \in[-V, V]$, and $\bar{\varphi}(x)$ if $x \in$ $(-\infty, V-1] \cup[V+1, \infty) ;$ and $\bar{\varphi}_{\kappa}^{1}(x)=\varphi_{\kappa}^{1}(x)$, if $x \in[-V, V]$, and $\bar{\varphi}(x)$ if $x \in(-\infty,-V$ $-1] \cup[V+1, \infty)$.

Below the configuration $\bar{\varphi}_{\kappa}^{1}(x)$ defined for usual contours will be denoted by $\bar{\varphi}_{\kappa}(x)$.

The weights of the usual contour $K$ and interface contour $I K$ will be calculated by the following formulas:

$$
\begin{gathered}
\gamma(K)=H\left(\psi_{K}(x)\right)-H\left(\bar{\varphi}_{\kappa}(x)\right), \\
\gamma(I K)=H\left(\psi_{I K}(x)\right)-H\left(\bar{\varphi}_{\kappa}^{1}(x)\right) .
\end{gathered}
$$

The proof of Theorem 4 is based on the following idea. Let the boundary conditions $\bar{\varphi}(x)$ $=[\varphi(x), x \in(-\infty,-V-1] \cup[V+1, \infty)]$ be fixed. The set of all configurations $\varphi(x) ; x \in$ $[-V, V]$ we denote via $\Phi(V)$. Suppose a configuration $\varphi_{\min }(x) \in \Phi(V)$ be a configuration with the minimal energy:

$$
H\left(\varphi_{\min }(x)\right)=\min _{\varphi(x) \in \Phi(V)} H(\varphi(x)) .
$$

Then the configuration $\varphi_{\min }(x)$ almost coincides with a special ground state of the model (1) (Lemma 1 in Sec. II). This fact allows us, based on special ground states, to define a common (for all boundary conditions) contour model and after that by using well-known trick ${ }^{14}$ (this trick, which was introduced in Ref. 14 for some special extensions of Pirogov-Sinai theory, is directly applicable to one-dimensional models with long-range interaction) to come to noninteracting clusters from interacting contours. Consider an arbitrary segment $I$, a sufficiently large volume $V$, two arbitrary boundary conditions $\varphi^{1}(x)$ and $\varphi^{2}(x)$. It turns out that the dependence of the expression $\mathbf{P}^{\mathbf{1}}\left(\varphi^{\prime}(I)\right) / \mathbf{P}^{2}\left(\varphi^{\prime}(I)\right)$ on the boundary conditions $\varphi^{1}(x)$ and $\varphi^{2}(x)$ can be estimated through the sum of statistical weights of super clusters connecting the segment $I$ with the boundary and this sum is negligible. Thus, two arbitrary extreme Gibbs states are relatively continuous and hence coincide. In Ref. 13 we developed this method [the estimation of dependence of the expression $\mathbf{P}^{1}\left(\varphi^{\prime}(I)\right) / \mathbf{P}^{2}\left(\varphi^{\prime}(I)\right)$ on the boundary conditions through the sum of statistical weights of super clusters connecting the segment $I$ with the boundary] at low temperatures. It turns out that after some modification the method works at all temperatures.

The contents of this paper are as follows. In Sec. II we prove Theorem 4, in Sec. III we complete the proof of Theorem 1 .

\section{UNIQUENESS OF GIBBS STATES: THE DENSITY $\kappa$ IS $p / q$}

Let us now introduce some necessary facts. 
Suppose that the value of the external field $\mu$ of the model (1) belongs to the interval $\left(\mu_{\kappa}^{-}, \mu_{\kappa}^{+}\right)$for some number $\kappa=q / p$.

Let the boundary conditions $\varphi^{1}(x)=\left[\varphi^{1}(x), x \in(-\infty,-V-1] \cup[V+1, \infty)\right]$ be fixed and

$$
\begin{aligned}
H\left(\varphi(x) \mid \varphi^{1}(x)\right)= & -\mu \sum_{x \in \mathbf{Z}^{1}, x \in[-V, V]} \varphi(x)+\sum_{x, y \in \mathbf{Z}^{1}, x>y ; x, y \in[-V, V]} U(x-y) \varphi(x) \varphi(y) \\
& +\sum_{x, y \in \mathbf{Z}^{1}, x>y ; x \in[-V, V] ; y \notin[-V, V]} U(x-y) \varphi(x) \varphi^{1}(y) \\
& +\sum_{x, y \in \mathbf{Z}^{1}, x>y ; x \notin[-V, V], y \in[-V, V]} U(x-y) \varphi^{1}(x) \varphi(y) .
\end{aligned}
$$

Lemma 1: Let $\varphi_{\min }(x) \in \Phi(V)$ be a configuration with the minimal energy:

$$
H\left(\varphi_{\min }(x) \mid \varphi^{1}(x)\right)=\min _{\varphi(x) \in \Phi(V)} H\left(\varphi(x) \mid \varphi^{1}(x)\right) .
$$

Then the configuration $\varphi_{\min }(x)$ has the following structure.

The restriction of the configuration $\varphi_{\min }(x)$ on the set $\left[-V+N_{b}, V-N_{b}\right]$ contains at most $p-1$ contours, moreover, all of them are interface contours $I K_{i,}, i=1, \ldots, m$, where $m<p-1$ and $\left|\operatorname{supp} I K_{i}\right|<3 d_{0} p+N_{b}$.

Lemma 1 was proved in Ref. 13 [see Lemma 12 (Ref. 13) and Sec. 5 of Ref. 13].

Let $H\left(\varphi(x) \mid \varphi^{1}(x), \varphi_{\min }(x)\right)$ denote the relative energy of a configuration $\varphi(x)$ [with respect to $\left.\varphi_{\min }(x)\right]:$

$$
H\left(\varphi(x) \mid \varphi^{1}(x), \varphi_{\min }(x)\right)=H\left(\varphi(x) \mid \varphi^{1}(x)\right)-H\left(\varphi_{\min }(x) \mid \varphi^{1}(x)\right) .
$$

Consider the Gibbs distribution $\mathbf{P}^{1}$ on $\Phi(V)$ corresponding to the boundary conditions $\varphi^{1}(x)=\left[\varphi^{1}(x), x \in(-\infty,-V-1] \cup[V+1, \infty)\right]$ :

$$
\mathbf{P}^{1}\left(\varphi^{\prime}(x)\right)=\frac{\exp \left(-\beta\left(H\left(\varphi^{\prime}(x) \mid \varphi^{1}(x), \varphi_{\min }(x)\right)\right)\right)}{\sum_{\varphi(x) \in \Phi(V)} \exp \left(-\beta\left(H\left(\varphi(x) \mid \varphi^{1}(x), \varphi_{\min }(x)\right)\right)\right)} .
$$

Let $\varphi(x) \in \Phi(V)$ be an arbitrary configuration, the boundary of the $\varphi(x)$ includes a finite number of usual contours $K_{i} ; i=1, \ldots, n$, and a finite number of interface contours $I K_{i} ; i=n$ $+1, \ldots, n+m$. Let $K_{i}=K_{i} ; i=1, \ldots, n ; K_{i}=I K_{i} ; i=n+1, \ldots, n+m$. The set of all contours of the boundary conditions $\varphi^{1}(x)$ will be denoted by $K_{0}$.

The statistical weights of contours and interface contours are

$$
w\left(K_{i}\right)=\exp \left(-\beta \gamma\left(K_{i}\right)\right) .
$$

The following equation is a direct consequence of the formulas (3), (4), and (7)

$$
\exp \left(-\beta H\left(\varphi(x) \mid \varphi^{1}(x), \varphi_{\min }(x)\right)\right)=Q_{1} \prod_{i=1}^{n+m} w\left(K_{i}\right) \exp \left(-\beta G\left(K_{0}, K_{1}, \ldots, K_{n+m}\right),\right.
$$

where the multiplier $G\left(K_{0}, K_{1}, \ldots, K_{n+m}\right)$ corresponds to the interaction between contours (usual and interface), and with the boundary conditions $\varphi^{1}(x)$

$$
G\left(K_{0}, K_{1}, \ldots, K_{n+m}\right)=\sum_{i, j=0 ; i<j}^{n+m} G\left(K_{i}, K_{j}\right)=\sum_{i, j ; i<j} \sum_{(x, y) \in \operatorname{Int}\left(K_{i}, K_{j}\right)} f(x, y, \varphi)
$$

and the multiplier $Q_{1}=Q_{1}\left(V, \varphi(x), \varphi^{1}(x)\right)$ is uniformly bounded from below and above: 0 $<$ const $_{1}<Q_{1}<$ const $_{2}$. The factor $Q_{1}$ appears due to the facts that the configuration $\varphi_{\min }(x)$ not necessarily coincides with a special ground state and is bounded due to Lemma 1. 
Now we write down the value of the interaction between the contours $K_{i}$ and $K_{j}$, the value of the interaction between the interface contours $I K_{i}$ and $I K_{j}$ and the value of the interaction between contour $K_{i}$ and interface contour $I K_{j}$.

Suppose $\operatorname{supp} K_{l}=\left[a_{l}, b_{l}\right] ; \operatorname{supp} I K_{l}=\left[a_{l}, b_{l}\right]$.

Let

$$
\operatorname{supp} I K_{i}^{+}=\left[b_{i}, a_{i+1}\right] \text { and } \operatorname{supp} I K_{i}^{-}=\left[b_{i-1}, a_{i}\right] \text {, }
$$

where $b_{0}=c$, if there exists $K \in B\left(\varphi^{\prime}(x)\right)$, such that $\operatorname{supp} K=[-\infty, c]$ and $b_{0}=-\infty$ otherwise; $a_{m+1}=d$, if there exists $K \in B\left(\varphi^{\prime}(x)\right)$, such that supp $K=[d, \infty]$ and $a_{m+1}=\infty$ otherwise.

(1) The contour $K_{i} \in B\left(\varphi^{\prime}(x)\right)$ interacts with the contour $K_{j} \in B\left(\varphi^{\prime}(x)\right)$ through all pairs $(x, y)$, such that $(x, y) \in \operatorname{Int}\left(K_{i}, K_{j}\right)$ and $f^{\prime}(x, y, \varphi) \neq 0$ where

$$
\operatorname{Int}\left(K_{i}, K_{j}\right)=\left[(x, y): x, y \in \mathbf{Z}^{1} ; x \in \operatorname{supp} K_{i}, y \in \operatorname{supp} K_{j}\right] .
$$

The value of the interaction

$$
\begin{aligned}
f^{\prime}(x, y, \varphi)= & U(x-y)\left(\varphi^{\prime}(x) \varphi^{\prime}(y)-\psi_{K_{i}}(x) \psi_{K_{i}}(y)+\bar{\varphi}_{\kappa}^{i}(x) \bar{\varphi}_{\kappa}^{i}(y)\right. \\
& \left.-\psi_{K_{j}}(x) \psi_{K_{j}}(y)+\bar{\varphi}_{\kappa}^{j}(x) \bar{\varphi}_{\kappa}^{j}(y)\right) .
\end{aligned}
$$

(2) The interface contour $I K_{i} \in B\left(\varphi^{\prime}(x)\right)$ interacts with the interface contour $I K_{j}$ $\in B\left(\varphi^{\prime}(x)\right)$ (let $\left.a_{j}>b_{i}\right)$ through all pairs $(x, y)$, such that $(x, y) \in \operatorname{Int}\left(I K_{i}, I K_{j}\right)$ and $f^{\prime \prime}(x, y) \neq 0$, where

$$
\begin{gathered}
\operatorname{Int}\left(I K_{i}, I K_{j}\right)=\operatorname{Int}^{1}\left(I K_{i}, I K_{j}\right)+\operatorname{Int}^{2}\left(I K_{i}, I K_{j}\right)+\operatorname{Int}^{3}\left(I K_{i}, I K_{j}\right)+\operatorname{Int}^{4}\left(I K_{i}, I K_{j}\right), \\
\operatorname{Int}^{1}\left(I K_{i}, I K_{j}\right)=\left[(x, y): x, y \in \mathbf{Z}^{1} ; x \in \operatorname{supp} I K_{i} \text { and } y \in \operatorname{supp} I K_{j}\right], \\
\operatorname{Int}^{2}\left(I K_{i}, I K_{j}\right)=\left[(x, y): x, y \in \mathbf{Z}^{1} ; x \in \operatorname{supp} I K_{i} \text { and } y \in \operatorname{supp} I K_{j}^{+}\right], \\
\operatorname{Int}^{3}\left(I K_{i}, I K_{j}\right)=\left[(x, y): x, y \in \mathbf{Z}^{1} ; x \in \operatorname{supp} I K_{i}^{-} \quad \text { and } y \in \operatorname{supp} I K_{j}\right], \\
\operatorname{Int}^{4}\left(I K_{i}, I K_{j}\right)=\left[(x, y): x, y \in \mathbf{Z}^{1} ; x \in \operatorname{supp} I K_{i}^{-} \quad \text { and } y \in \operatorname{supp} I K_{j}^{+}\right] .
\end{gathered}
$$

The value of the interaction

$$
\begin{aligned}
f^{\prime \prime}(x, y, \varphi)=f_{1}^{\prime \prime}(x, y)= & U(x-y)\left(\varphi^{\prime}(x) \varphi^{\prime}(y)-\psi_{I K_{i}}(x) \psi_{I K_{i}}(y)\right. \\
& \left.+\bar{\varphi}_{\kappa}^{i}(x) \bar{\varphi}_{\kappa}^{i}(y)-\psi_{I K_{j}}(x) \psi_{I K_{j}}(y)+\bar{\varphi}_{\kappa}^{j}(x) \bar{\varphi}_{\kappa}^{j}(y)\right)
\end{aligned}
$$

if $(x, y) \in \operatorname{Int}^{2}\left(I K_{i}, I K_{j}\right)$,

$$
f^{\prime \prime}(x, y)=f_{2}^{\prime \prime}(x, y)=U(x-y)\left(\varphi^{\prime}(x) \varphi^{\prime}(y)-\psi_{I K_{i}}(x) \psi_{I K_{i}}(y)+\bar{\varphi}_{\kappa}^{i}(x) \bar{\varphi}_{\kappa}^{i}(y)\right)
$$

if $(x, y) \in \operatorname{Int}^{2}\left(I K_{i}, I K_{j}\right)$,

$$
\left.f^{\prime \prime}(x, y)=f_{3}^{\prime \prime}(x, y)=U(x-y)\left(\varphi^{\prime}(x) \varphi^{\prime}(y)-\psi_{I K_{j}}(x) \psi_{I K_{j}}(y)\right)+\bar{\varphi}_{\kappa}^{j}(x) \bar{\varphi}_{\kappa}^{j}(y)\right)
$$

if $(x, y) \in \operatorname{Int}^{3}\left(I K_{i}, I K_{j}\right)$,

$$
f^{\prime \prime}(x, y)=f_{4}^{\prime \prime}(x, y)=U(x-y)\left(\varphi^{\prime}(x) \varphi^{\prime}(y)-\bar{\varphi}_{\kappa}^{1, i}(x) \bar{\varphi}_{\kappa}^{1, i}(y)-\bar{\varphi}_{\kappa}^{2, j}(x) \bar{\varphi}_{\kappa}^{2, j}(y)\right)
$$

if $(x, y) \in \operatorname{Int}^{4}\left(I K_{i}, I K_{j}\right)$. 
(3) The contour $K_{i} \in B\left(\varphi^{\prime}(x)\right)$ interacts with the interface contour $I K_{j} \in B\left(\varphi^{\prime}(x)\right)$ through all pairs $(x, y)$, such that $(x, y) \in \operatorname{Int}\left(K_{i}, I K_{j}\right)$ and $f^{\prime \prime \prime}(x, y) \neq 0$, where

$$
\begin{gathered}
\operatorname{Int}\left(K_{i}, I K_{j}\right)=\operatorname{Int}^{1}\left(K_{i}, I K_{j}\right)+\operatorname{Int}^{2}\left(K_{i}, I K_{j}\right), \\
\operatorname{Int}^{1}\left(K_{i}, I K_{j}\right)=\left[(x, y): x, y \in \mathbf{Z}^{1} ; x \in \operatorname{supp} K_{i} \quad \text { and } y \in \operatorname{supp} I K_{j}\right], \\
\operatorname{Int}^{2}\left(K_{i}, I K_{j}\right)=\left[(x, y): x, y \in \mathbf{Z}^{1} ; x \in \operatorname{supp} K_{i} \quad \text { and } y \in \operatorname{supp} I K_{j}^{+}\right]
\end{gathered}
$$

if $a_{j}>b_{i}$, and

$$
\operatorname{Int}^{2}\left(K_{i}, I K_{j}\right)=\left[(x, y): x, y \in \mathbf{Z}^{1} ; x \in \operatorname{supp} K_{i} \quad \text { and } y \in \operatorname{supp} I K_{j}^{-}\right]
$$

if $a_{i}>b_{j}$.

The value of the interaction

$$
\begin{aligned}
f^{\prime \prime \prime}(x, y)=f_{1}^{\prime \prime \prime}(x, y)= & U(x-y)\left(\varphi^{\prime}(x) \varphi^{\prime}(y)-\psi_{K_{i}}(x) \psi_{K_{i}}(y)\right. \\
& \left.\left.+\bar{\varphi}_{\kappa}^{i}(x) \bar{\varphi}_{\kappa}^{i}(y)-\psi_{I K_{j}}(x) \psi_{I K_{j}}(y)\right)+\bar{\varphi}_{\kappa}^{j}(x) \bar{\varphi}_{\kappa}^{j}(y)\right)
\end{aligned}
$$

if $(x, y) \in \operatorname{Int}^{1}\left(K_{i}, I K_{j}\right)$,

$$
f^{\prime \prime \prime}(x, y)=f_{2}^{\prime \prime \prime}(x, y)=U(x-y)\left(\varphi^{\prime}(x) \varphi^{\prime}(y)-\psi_{K_{i}}(x) \psi_{K_{i}}(y)+\bar{\varphi}_{\kappa}^{i}(x) \bar{\varphi}_{\kappa}^{i}(y)\right)
$$

if $(x, y) \in \operatorname{Int}^{2}\left(K_{i}, I K_{j}\right)$.

For simplicity $K_{i}, i=1, \ldots, n+m$ will be denoted by $K_{i}, i \in$ Ind, where the statistical weights $w\left(K_{i}\right)$ are defined by the formulas (7), (3), and (4). Thus, the formula (8) has the form

$$
\exp \left(-\beta H\left(\varphi(x) \mid \varphi^{1}(x), \varphi_{\min }(x)\right)\right)=Q_{1} \prod_{i \in \operatorname{Ind}} w\left(K_{i}\right) \exp \left(-\beta G\left(K_{0}, K_{1}, \ldots, K_{n+m}\right)\right) .
$$

The set of all pairs $(x, y)$ in the double sum (9) will be denoted by $Y=Y\left(K_{0}, K_{1}, \ldots, K_{n+m}\right)$. Write (10) as follows:

$$
\exp \left(-\beta H\left(\varphi(x) \mid \varphi^{1}(x), \varphi_{\min }(x)\right)\right)=Q_{1} \prod_{i \in \operatorname{Ind}} w\left(K_{i}\right) \prod_{(x, y) \in Y}(1+\exp (-\beta f(x, y, \varphi)-1) .
$$

From (11) we get

$$
\exp \left(-\beta H\left(\varphi(x) \mid \varphi^{1}(x), \varphi_{\min }(x)\right)\right)=Q_{1} \prod_{G^{\prime} \subset G} \prod_{i \in \operatorname{Ind}} w\left(K_{i}\right) \prod_{(x, y) \in Y^{\prime} ; f(x, y, \varphi) \neq 0} g(x, y),
$$

where the summation is taken over all subsets $Y^{\prime}$ (including the empty set) of the set $Y$, and $g(x, y, \varphi)=\exp (-\beta f(x, y, \varphi))-1$.

Consider an arbitrary term of the sum (12), which corresponds to the subset $Y^{\prime} \subset Y$. Let the bond $(x, y) \in Y^{\prime}$. Below, contours and interface contours will be called contours. Consider the set $\mathbf{K}$ of all contours such that for each contour $K \subset \mathbf{K}$, the set supp $K \cap(x \cup y)$ contains one point. We call any two contours from $\mathbf{K}$ connected. The set of contours $\mathbf{K}$ is called $Y^{\prime}$ connected if for any two contours $K_{a}$ and $K_{b}$ there exists a collection $\left(K_{1}=K_{a}, K_{2}, \ldots, K_{n}=K_{b}\right)$ such that any two contours $K_{i}$ and $K_{i+1}, i=1, \ldots, n-1$, are connected by some bond $(x, y) \in Y^{\prime}$.

The pair $D=\left[\left(K_{i}, i=1, \ldots, s\right) ; Y^{\prime}\right]$, where $Y^{\prime}$ is some set of bonds, is called a cluster provided there exists a configuration $\varphi(x)$ such that $K_{i} \in B(\varphi(x)) ; i=1, \ldots, s ; Y^{\prime} \subset Y$; and the set $\left(K_{i}, i\right.$ $=1, \ldots, S)$ is $Y^{\prime}$ connected. The statistical weight of a cluster $D$ is defined by the formula. 


$$
w(D)=\prod_{i=1}^{s} w\left(K_{i}\right) \prod_{(x, y) \in Y^{\prime}} g(x, y, \varphi) .
$$

Two clusters $D_{1}$ and $D_{2}$ are called compatible provided any two contours $K_{1}$ and $K_{2}$ belonging to $D_{1}$ and $D_{2}$, respectively, are compatible and not connected. A set of clusters is called compatible provided any two clusters of it are compatible.

If $D=\left[\left(K_{i}, i=1, \ldots, s\right) ; Y^{\prime}\right]$, then we say that $K_{i} \in D ; i=1, \ldots, s$.

The following lemma is a direct consequence of the definitions.

Lemma 2: Let the boundary conditions $\varphi^{1}(x)=\left[\varphi^{1}(x), x \in(-\infty,-V-1] \cup[V+1, \infty)\right]$ be fixed.

If $\left[D_{1}, \ldots, D_{m}\right]$ is a compatible set of clusters and $\cup_{i=1}^{m} \operatorname{supp} D_{i} \subset[-V, V]$, then there exists a configuration $\varphi(x)$ which contains this set of clusters. For each configuration $\varphi(x)$ we have

$$
\exp \left(-\beta H\left(\varphi(x) \mid \varphi^{1}(x), \varphi_{\min }(x)\right)\right)=Q_{1} \sum_{Y^{\prime} \subset Y} \prod w\left(D_{i}\right)
$$

where the clusters $D_{i}$ are completely determined by the set $Y^{\prime}$. The partition function is

$$
\Xi\left(\varphi^{1}(x)\right)=Q \sum w\left(D_{1}\right) \cdots w\left(D_{m}\right),
$$

where the summation is taken over all nonordered compatible collections of clusters and the factor $Q=Q\left(V, \varphi^{1}(x)\right)$ is uniformly bounded: $0<$ const $<Q<$ const $_{2}$.

Lemma 2 shows that we come to noninteracting clusters from interacting contours.

Let $\mathbf{P}^{1}$ and $\mathbf{P}^{2}$ be two Gibbs states of the model (1) corresponding to the boundary conditions $\varphi^{1}(x)$ and $\varphi^{2}(x)$, respectively.

The following lemma has a key role in the proof of Theorem 4.

Lemma 3: Suppose that the value of the external field $\mu$ of the model (1) belongs to the interval $\left(\mu_{\kappa}^{-}, \mu_{\kappa}^{+}\right)$for some number $\kappa=q / p$.

Then the measures $\mathbf{P}^{1}$ and $\mathbf{P}^{2}$ are absolutely continuous with respect to each other.

Proof: Let $I=[a, b]$ be an arbitrary segment and $\varphi^{\prime}(I)$ be an arbitrary configuration.

In order to prove the lemma we show that there exist two positive constants $s$ and $S$ not depending on $I, \varphi^{1}(x), \varphi^{2}(x)$ and $\varphi^{\prime}(I)$, such that

$$
s \leqslant \mathbf{P}^{1}\left(\varphi^{\prime}(I)\right) / \mathbf{P}^{2}\left(\varphi^{\prime}(I)\right) \leqslant S .
$$

Let $\mathbf{P}_{\mathbf{V}}^{1}$ and $\mathbf{P}_{\mathbf{V}}^{2}$ be Gibbs measures corresponding to the boundary conditions $\varphi^{1}(x)$, and $\varphi^{2}(x), x \in \mathbf{Z}^{1}-I_{V}$, respectively, where $I_{V}=[-V, V]$.

Therefore,

$$
\lim _{V \rightarrow \infty} \mathbf{P}_{\mathbf{V}}^{1}=\mathbf{P}^{1} \quad \text { and } \lim _{V \rightarrow \infty} \mathbf{P}_{\mathbf{V}}^{2}=\mathbf{P}^{2}
$$

where by convergence we mean weak convergence of probability measures.

In order to establish the inequality (14) it will be proved that for each fixed interval $I$, $I \subset[-M, M]$ there exists a number $V_{0}(M)$, which depends on $M$ only, such that

$$
s \leqslant \mathbf{P}_{\mathbf{V}}^{1}\left(\varphi^{\prime}(I)\right) / \mathbf{P}_{\mathbf{V}}^{2}\left(\varphi^{\prime}(I)\right) \leqslant S
$$

if $V>V_{0}$.

Consider 


$$
\begin{aligned}
\mathbf{P}_{\mathbf{V}}^{1}\left(\varphi^{\prime}(I)\right) & =\frac{\sum_{\varphi\left(I_{V}\right): \varphi(I)=\varphi^{\prime}(I)} \exp \left(-\beta H\left(\varphi\left(I_{V}\right) \mid \varphi^{1}(x), \varphi_{\min }(x)\right)\right) O\left(\varphi(I), V, \varphi^{1}\right)}{\sum_{\varphi\left(I_{V}\right)} \exp \left(-\beta H\left(\varphi\left(I_{V}\right) \mid \varphi^{1}(x), \varphi_{\min }(x)\right)\right) O\left(\varphi(I), V, \varphi^{1}\right)} \\
& =\frac{\Xi\left(I_{V}-I \mid \varphi^{1}(x), \varphi^{\prime}(I), \varphi_{\min }(x)\right) O\left(\varphi(I), V, \varphi^{1}\right)}{\sum_{\varphi^{\prime \prime}(I)} \Xi\left(I_{V}-I \mid \varphi^{1}(x), \varphi^{\prime \prime}(I), \varphi_{\min }(x)\right) O\left(\varphi(I), V, \varphi^{1}\right)}
\end{aligned}
$$

where $\Xi\left(I_{V}-I \mid \varphi^{1}(x), \varphi^{\prime}(I), \varphi_{\min }(x)\right)$ denotes the partition function corresponding to the boundary conditions $\varphi^{1}(x), x \in \mathbf{Z}^{1}-I_{V}, \varphi^{\prime}(I), x \in I$ and

$$
O\left(\varphi(I), V, \varphi^{1}\right)=\exp \left(-\beta \sum_{x, y \in \mathbf{Z}^{1} ; x \in \mathbf{Z}^{1}-I_{V}, y \in I} U(x-y)\left(\varphi^{1}(x) \varphi(y)-\varphi^{1}(x) \varphi_{\min }(x)\right)\right) .
$$

We can express $\mathbf{P}_{\mathbf{V}}^{2}\left(\varphi^{\prime}(I)\right)$ in just the same way. (17):

In order to prove the inequality (15) it is enough to establish inequality (16) and inequality

$$
1 / 2<O\left(\varphi(I), V, \varphi^{i}(x)\right)<2, \quad i=1,2
$$

[where the inequalities in (16) are held uniformly with respect to $\varphi(I)$ and $\varphi^{i}$ : for each $I$ there exists $V$, not depending on $\varphi(I)$ and $\left.\varphi^{i}\right]$ and

$$
1 / S \leqslant \frac{\Xi\left(I_{V}-I \mid \varphi^{1}(x), \varphi^{\prime \prime}(I), \varphi_{\min }(x)\right)}{\Xi\left(I_{V}-I \mid \varphi^{1}(x), \varphi^{\prime}(I), \varphi_{\min }(x)\right)} / \frac{\Xi\left(I_{V}-I \mid \varphi^{2}(x), \varphi^{\prime \prime}(I), \varphi_{\min }(x)\right)}{\Xi\left(I_{V}-I \mid \varphi^{2}(x), \varphi^{\prime}(I), \varphi_{\min }(x)\right)} \leqslant 1 / s
$$

for arbitrary $\varphi^{\prime \prime}(I)$.

Indeed, if the inequality (17) holds, then

$$
\begin{aligned}
& \frac{\Xi\left(I_{V}-I \mid \varphi^{1}(x), \varphi^{\prime}(I), \varphi_{\min }(x)\right)}{\sum_{\varphi^{\prime \prime}(I)} \Xi\left(I_{V}-I \mid \varphi^{1}(x), \varphi^{\prime \prime}(I), \varphi_{\min }(x)\right)} / \frac{\Xi\left(I_{V}-I \mid \varphi^{2}(x), \varphi^{\prime}(I), \varphi_{\min }(x)\right)}{\sum_{\varphi^{\prime \prime}(I)} \Xi\left(I_{V}-I \mid \varphi^{2}(x), \varphi^{\prime \prime}(I), \varphi_{\min }(x)\right)} \\
& =\mathbf{A}_{\mathbf{V}}^{\mathbf{1}}\left(\varphi^{\prime}(I)\right) / \mathbf{A}_{\mathbf{V}}^{\mathbf{2}}\left(\varphi^{\prime}(I)\right) \\
& =1 /\left(\frac{\sum_{\varphi^{\prime \prime}(I)} \Xi\left(I_{V}-I \mid \varphi^{1}(x), \varphi^{\prime \prime}(I), \varphi_{\min }(x)\right)}{\Xi\left(I_{V}-I \mid \varphi^{1}(x), \varphi^{\prime}(I), \varphi_{\min }(x)\right)} / \frac{\sum_{\varphi^{\prime \prime}(I)} \Xi\left(I_{V}-I \mid \varphi^{2}(x), \varphi^{\prime \prime}(I), \varphi_{\min }(x)\right)}{\Xi\left(I_{V}-I \mid \varphi^{2}(x), \varphi^{\prime}(I), \varphi_{\min }(x)\right)}\right) \\
& =1 / \frac{\left(\sum_{\varphi^{\prime \prime}(I)} \Xi\left(I_{V}-I \mid \varphi^{1}(x), \varphi^{\prime \prime}\left(I, \varphi_{\min }(x)\right)\right) \Xi\left(I_{V}-I \mid \varphi^{2}(x), \varphi^{\prime}(I), \varphi_{\min }(x)\right)\right.}{\left(\sum_{\varphi^{\prime \prime}(I)} \Xi\left(I_{V}-I \mid \varphi^{2}(x), \varphi^{\prime \prime}(I), \varphi_{\min }(x)\right)\right) \Xi\left(I_{V}-I \mid \varphi^{1}(x), \varphi^{\prime}(I), \varphi_{\min }(x)\right)} .
\end{aligned}
$$

Therefore,

$$
1 /(1 / s) \leqslant \mathbf{A}_{\mathbf{V}}^{\mathbf{1}}\left(\varphi^{\prime}(I)\right) / \mathbf{A}_{\mathbf{V}}^{2}\left(\varphi^{\prime}(I)\right) \leqslant 1 /(1 / S)
$$

since the quotient of $\sum_{i=1}^{n} a_{i} / \sum_{i=1}^{n} b_{i}$ lies between $\min \left(a_{i} / b_{i}\right)$ and $\max \left(a_{i} / b_{i}\right)$.

Thus, if in addition, the inequality (16) holds, then

$$
2^{-4} s<\mathbf{P}_{\mathbf{V}}^{1}\left(\varphi^{\prime}(I)\right): \mathbf{P}_{\mathbf{V}}^{2}\left(\varphi^{\prime}(I)\right)<2^{4} S .
$$

Now we start to prove the inequalities (16) and (17).

It can be easily shown that (16) is a direct consequence of the condition $U(x) \sim A x^{-\gamma}$, at $x$ $\rightarrow \infty$; where $\gamma>1$, and $A$ is a strong positive constant.

So, in order to complete the proof of Lemma 3 we must establish the following inequality [which is just transformed inequality (17)]: 


$$
1 / S \leqslant \frac{\left.\Xi\left(I_{V}-I \mid \varphi^{1}(x), \varphi^{\prime \prime}(I), \varphi_{\min }(x)\right)\right) \Xi\left(I_{V}-I \mid \varphi^{2}(x), \varphi^{\prime}(I), \varphi_{\min }(x)\right)}{\left.\Xi\left(I_{V}-I \mid \varphi^{2}(x), \varphi^{\prime \prime}(I), \varphi_{\min }(x)\right)\right) \Xi\left(I_{V}-I \mid \varphi^{1}(x), \varphi^{\prime}(I), \varphi_{\min }(x)\right)}=\frac{\Xi^{1, "} \Xi^{2,,^{\prime}}}{\Xi^{2,{ }^{\prime \prime}} \Xi^{1,}} \leqslant 1 / s .
$$

Consider

$$
\Xi^{1,{ }^{\prime \prime}} \Xi^{2,}{ }^{\prime}=\Xi\left(I_{V}-I \mid \varphi^{1}(x), \varphi^{\prime \prime}(I), \varphi_{\min }(x)\right) \Xi\left(I_{V}-I \mid \varphi^{2}(x), \varphi^{\prime}(I), \varphi_{\min }(x)\right) .
$$

The following generalization of the definition of the compatibility allows us to represent $\Xi^{1, "} \Xi^{2,}$ as a single partition function.

A set of clusters is called super compatible provided any of its two parts coming from two partitions sums is compatible. In other words, in super compatibility an intersection of supports of two clusters is allowed.

The following lemma is an analogue of Lemma 2.

Lemma 4: Let boundary conditions $\varphi^{1}(x)=\left[\varphi^{1}(x), x \in(-\infty,-V-1] \cup[V+1, \infty)\right]$ and $\varphi^{2}(x)=\left[\varphi^{2}(x), x \in(-\infty,-V-1] \cup[V+1, \infty)\right]$ be fixed.

If $\left[D_{1}, \ldots, D_{m}\right]$ is a super compatible set of clusters and $\cup_{i=1}^{m} \operatorname{supp} D_{i} \subset[-V, V]$, then there exist two configurations $\varphi^{3}(x)$ and $\varphi^{4}(x)$ which contain this set of clusters. For each two configurations $\varphi^{3}(x)$ and $\varphi^{4}(x)$ we have

$$
\exp \left(-\beta H\left(\varphi^{3}(x) \mid \varphi^{1}(x), \varphi_{\min }(x)\right) \exp \left(-\beta H\left(\varphi^{4}(x) \mid \varphi^{1}(x), \varphi_{\min }(x)\right)=Q_{1} \sum_{G^{\prime} \subset G, G^{\prime \prime} \subset G} \prod w\left(D_{i}\right),\right.\right.
$$

where the clusters $D_{i}$ are completely determined by the sets $G^{\prime}$ and $G^{\prime \prime}$. The super partition function is

$$
\Xi^{1, ",},,^{\prime}=\Xi^{1,{ }^{\prime \prime}} \Xi^{2,{ }^{\prime}}=Q \sum w\left(D_{1}\right) \cdots w\left(D_{m}\right),
$$

where the summation is taken over all nonordered super compatible collections of clusters and the factor $Q=Q\left(V, \varphi^{1}(x), \varphi^{2}(x)\right)$ is uniformly bounded: $0<$ const $_{1}<Q<$ const $_{2}$.

Lemma 4 is a direct consequence of the definitions.

An arbitrary connected component of an arbitrary super compatible set of clusters will be called a super clusters. A super cluster $S D=\left[\left(K_{i}, i=1, \ldots, r\right) ; G^{\prime}\right]$ is said to be long if the intersection of the set $\left.\left(\cup_{i=1}^{m} \operatorname{supp} K_{i}\right)\right) \cup G^{\prime}$ with both $I$ and $\mathbf{Z}^{1}-I_{V}=(-\infty,-V-1] \cup[V+1, \infty)$ is nonempty. In other words, a long super cluster connects the boundary with the segment $I$.

A set of super clusters is called compatible provided the set of all clusters belonging to these super clusters are super compatible.

It turns out that in our estimates long super clusters are negligible.

Lemma 5: For each fixed interval I, there exists a number $V_{0}(I)$, which depends on I only, such that if $V>V_{0}(I)$

$$
1 / 2 \Xi^{1,{ }^{\prime}, 2, "}<\Xi^{1,{ }^{\prime}, 2, ",(n . l .)}=\sum w\left(S D_{1}\right) \cdots w\left(S D_{m}\right)<3 / 2 \Xi^{1,{ }^{\prime}, 2,{ }^{\prime \prime},}
$$

where the summation is taken over all nonlong, nonordered compatible collections of super clusters $\left[S D_{1}, \ldots, S D_{m}\right], \cup_{i=1}^{m} \operatorname{supp}\left(S D_{i}\right) \subset I_{N}-I$ corresponding to the boundary conditions $\varphi^{1}(x), \varphi^{2}(x), x \in \mathbf{Z}^{1}-I_{V} ; \varphi^{\prime}(x)$ and $\varphi^{\prime \prime}(x), x \in I$.

Consider a collection of contours $K_{0}, K_{1}, \ldots, K_{n}$. The value of the interaction of the contour $K_{0}$ with the contours $K_{1}, \ldots, K_{n}$ we denote by $G\left(K_{0} \mid K_{1}, \ldots, K_{n}\right)$ :

$$
G\left(K_{0} \mid K_{1}, \ldots, K_{n}\right)=\prod_{B \in I G(0 \mid 1, \ldots, n)}(1+\exp (-\beta f(B)-1)),
$$


where $I G(0 \mid 1, \ldots, n)$ is the set of all interaction elements intersecting the support of the contour $K_{0}$.

Lemma 6:

$$
\begin{aligned}
G\left(K_{0} \mid K_{1}, \ldots, K_{n}\right) & =\prod_{B \in I G(0 \mid 1, \ldots, n)} \mid(1+\exp (-\beta f(B)-1)) \\
& \leqslant \operatorname{const}(\operatorname{dist}(0 \mid 1, \ldots, n))^{-\alpha}\left(\left|\operatorname{supp}\left(K_{0}\right)\right|\right)^{1-\alpha},
\end{aligned}
$$

where $\operatorname{dist}(0 \mid 1, \ldots, n)$ is the distance between the support of $K_{0}$ and the union of the supports of contours $K_{1}, \ldots, K_{n}$.

In other words, the interaction of $K_{1}, \ldots, K_{n}$ on $K_{0}$ tends to zero when the distance between them increases, and value of the interaction increases with a rate less than the length of the support of $K_{0}$.

The technical Lemma 6 follows from the decreasing conditions of the potential $U(x)$. For the rigorous proof see Ref. 13, Lemma 4.

The following lemma is an analogue of Lemma 5 for clusters (not super clusters).

Lemma 7: For each fixed interval I, there exists a number $V_{0}(I)$, which depends on I only, such that if $V>V_{0}(I)$

$$
1 / 2 \Xi^{1,{ }^{\prime}}<\Xi^{1,{ }^{\prime},(n . l .)}=\sum w\left(D_{1}\right) \ldots w\left(D_{m}\right)<3 / 2 \Xi^{1,{ }^{\prime}},
$$

where the summation is taken over all nonlong, nonordered compatible collections of clusters $\left[D_{1}, \ldots, D_{m}\right], \cup_{i=1}^{m} \operatorname{supp} D_{i} \subset I_{N}-I$ corresponding to the boundary conditions $\varphi^{1}(x), x \in \mathbf{Z}^{1}$ $-I_{V} ; \varphi^{\prime}(x), x \in I$.

Proof:

$$
\Xi^{1,{ }^{\prime}}=\Xi^{1,{ }^{\prime},(n . l .)}+\left(\Xi^{1,{ }^{\prime}}-\Xi^{1{ }^{\prime},(n . l .)}\right)=\Xi^{1,{ }^{\prime},(n . l .)}+\Xi^{1{ }^{\prime},(l .)},
$$

where the summation in $\Xi^{1,{ }^{\prime},(l .)}$ is taken over all nonordered compatible collections of clusters $\left[D_{1}, \ldots, D_{m}\right]$ containing at least one long cluster, $\cup_{i=1}^{m} \operatorname{supp} D_{i} \subset I_{N}-I$ corresponding to the boundary conditions $\varphi^{1}(x), x \in \mathbf{Z}^{1}-I_{V} ; \varphi^{\prime}(x), x \in I$.

By dividing both sides of the last equality by $\Xi^{1,{ }^{\prime}}$, we get

$$
1=\Xi^{1,{ }^{\prime},(n . l .)} / \Xi^{1,{ }^{\prime}}+\Xi^{1,{ }^{\prime},(l .)} / \Xi^{1,{ }^{\prime}} .
$$

Now we are going to show that the second term (which is not necessarily positive) is negligible, that is the absolute value of it is less than $1 / 2$ (actually we can show that the absolute value of the second term is less than any fixed positive number at sufficiently large values of $V$ ).

The term $\Xi^{1{ }^{\prime},(l .)} / \Xi^{1{ }^{\prime}}$ can be interpreted as a "probability" $P$ (Long) of the event that there exists at least one long cluster.

We show that the absolute value of this "probability" is less than $1 / 2$ by the following method. We estimate the density of long clusters: the probability that a given segment belongs to the support of some long cluster. Since some statistical weights of clusters are positive and some negative, we estimate the absolute values of these "probabilities." We show that for a fixed segment the "probability" that this segment belongs to the support of some long cluster with positive "probability" minus the "probability" that this segment belongs to the support of some long cluster with negative "probability" is less than one. Since the density is less than one, by the law of large numbers a "typical" long cluster has not very long support, and therefore has long bonds. When $V$ tends to infinity, the total length of bonds tends to infinity, and the impact of these bonds tends to zero.

Now we replace a statistical weight $w\left(D_{i}\right)$ of each cluster $D_{i}$ belonging to the configuration containing at least one long cluster with its absolute value (and "probability" of long cluster 
becomes positive) and the expression $\Xi^{1,{ }^{\prime},(l .)} / \Xi^{1,{ }^{\prime}}$ transfers into $\Xi^{1,{ }^{\prime},(l \text {.abs })} / \Xi^{\left.1,{ }^{\prime}, \text { (abs }\right)}$. It can be easily shown that, without loss of generality we can suppose that $\Xi^{1,{ }^{\prime},(l .)} \geqslant 0$. Obviously,

$$
\left|\Xi^{1, ',(l .)} / \Xi^{1{ }^{\prime}}\right| \leqslant \Xi^{1,{ }^{\prime},(l . \mathrm{abs})} / \Xi^{1,{ }^{\prime},(\mathrm{abs})} .
$$

Now the expression $\Xi^{1,},(l$ abs $) / \Xi^{1,}$, , (abs) can be interpreted as a "absolute probability" $P^{\text {abs }}$ (Long) of the event that there is at least one long cluster.

Now our aim is to estimate the "absolute probability" $P$ abs of the event that a given segment belongs to the support of long cluster. In other words, we are going to estimate the statistical weights of long clusters after replacing of the values of all negative bonds in configurations containing at least one long cluster with their absolute values.

Let $\varphi\left(I_{V}-I\right)$ be an arbitrary subconfiguration which contains contours $K_{1}, \ldots, K_{l}$, belonging to long clusters, $\mathbf{K}=\cup_{1}^{l} \operatorname{supp}^{1} K_{i}, \mathbf{K}^{\mathbf{1}}=\mathbf{K} \cap[-V,-(|I| / 2)]$ and $\mathbf{K}^{\mathbf{2}}=\mathbf{K} \cap[|I| / 2, V]$.

Put $C^{1}\left(\varphi\left(I_{V}-I\right)\right)=\left|\mathbf{K}^{1}\right|$ and $C^{2}\left(\varphi\left(I_{V}-I\right)\right)=\left|\mathbf{K}^{2}\right|$. We have

$$
\begin{aligned}
\mid P(\text { Long }) \mid & =\left|\Xi^{1,{ }^{\prime},(l .)} / \Xi^{1,{ }^{\prime}}\right| \\
& \leqslant P^{\mathrm{abs}}(\text { Long }) \\
& =\sum w^{\mathrm{abs}}\left(D_{1}\right) \cdots w\left(D_{m}\right) / \Xi^{1{ }^{\prime},{ }^{(\mathrm{abs})}} \\
& =\sum^{p, 1} w^{\mathrm{abs}}\left(D_{1}\right) \ldots w^{\mathrm{abs}}\left(D_{m}\right) / \Xi^{1,{ }^{\prime},(\mathrm{abs})}+\sum^{p, 2} w^{\mathrm{abs}}\left(D_{1}\right) \ldots w^{\mathrm{abs}}\left(D_{m}\right) / \Xi^{1,{ }^{\prime},(\mathrm{abs})} \\
& =P^{\mathrm{abs}}(\text { Long },>p)+P^{\mathrm{abs}}(\text { Long }, \leqslant p),
\end{aligned}
$$

where $w^{\text {abs }}\left(D_{i}\right)=\left|w\left(D_{i}\right)\right|$ for all clusters belonging to the configuration containing at least one long cluster and $w^{\text {abs }}\left(D_{i}\right)=w\left(D_{i}\right)$ for other clusters [note that the statistical weight $w^{\text {abs }}\left(D_{i}\right)$ of fixed cluster in one configuration can be positive, in other negative], last two summations are taken over all nonordered compatible collections of clusters $\left[D_{1}, \ldots, D_{m}\right]$ containing at least one long cluster, $\cup_{i=1}^{m} \operatorname{supp} D_{i} \subset I_{V}-I$ corresponding to the boundary conditions $\left\{\varphi^{1}(x), x \in \mathbf{Z}^{1}\right.$ $-I_{V} ; \varphi^{\prime}(x), x \in I_{\}}$, the summation in $\Sigma^{p, 1}$ is taken over all configurations $\varphi\left(I_{V}\right): \varphi(I)=\varphi^{\prime}(I)$; $2 C^{1}\left(\varphi\left(I_{V}-V\right)\right) /\left(\left|I_{V}\right|-|I|\right)>p ; 2 C^{2}\left(\varphi\left(I_{V}-V\right)\right) /\left(\left|I_{V}\right|-|I|\right)>p$, the summation in $\Sigma^{p, 2}$ is taken over all configurations $\quad \varphi\left(I_{V}\right): \varphi(I)=\varphi^{\prime}(I) ; \quad 2 C^{1}\left(\varphi\left(I_{V}-V\right)\right) /\left(\left|I_{V}\right|-|I|\right) \leqslant p ; \quad 2 C^{2}\left(\varphi\left(I_{V}\right.\right.$ $-V)) /\left(\left|I_{V}\right|-|I|\right) \leqslant p$. It means that the density of contours belonging to long clusters in each configuration from $\Sigma^{p, 1}\left(\Sigma^{p, 2}\right)$ in both segments $[-V,-(|I| / 2)]$ and $[|I| / 2, V]$ is greater than $p$ (is not greater than $p$ ).

We fixed the value of $p$ as $1-q / 2 l$, where the values of $q$ and $l$ will be defined in the proof of Lemma 9.

It turns out that the long clusters are negligible.

Lemma 8: For each fixed interval I there exists a value of $V_{0}$, such that if $V>V_{0}$

$$
P^{\text {abs }}(\text { Long })=P^{\text {abs }}(\text { Long, }>p)+P^{\text {abs }}(\text { Long, } \leqslant p)<1 / 2 \text {. }
$$

Lemma 8 is a consequence of the following two lemmas.

Lemma 9: For each fixed interval $I$ there exists a value of $V_{0}$, such that if $V>V_{0}$

$$
P^{\text {abs }}(\text { Long, }>p)<1 / 4 \text {. }
$$

Lemma 10: For each fixed interval $I$ there exists a value of $V_{0}$, such that if $V>V_{0}$

$$
P^{\mathrm{abs}}(\text { Long, } \leqslant p)<1 / 4 \text {. }
$$


Proof of Lemma 9: Consider the partition of $\mathbf{Z}^{1}$ into segments $T_{k}=T_{k}(l p)$, where $T_{k}(l p)$ is the segment with the center at $x=(l p / 2)+k l p$ and with the length $l p\left(T_{k}\right.$ consists of $l$ segments $I_{k}$ with the length $p$, where $p$ is the period of the special ground state). The value of $l$ will be defined later. Let us consider an arbitrary configuration $\varphi(x)$. We say that a segment $I_{k}$ is regular, if $I_{k}$ does not belong to the support of some long cluster. We say that a segment $T_{k}$ is super-regular, if $T_{k}$ contains at least one regular segment.

Let $\mathbf{P}_{\mathbf{V}}$ be a Gibbs measure corresponding to the boundary conditions $\varphi^{1}(x), x \in \mathbf{Z}^{\mathbf{1}}, \varphi^{\prime}(I)$, $x \in I$.

Let the segment $I_{V}-I$ consist of $n$ segments $T_{k} ; k=1, \ldots, n$.

We define a sample space $\Omega$ consisting of $2^{n}$ elementary events $A^{j}=[\sigma(1), \ldots, \sigma(n)]$, where $\sigma(k), k=1, \ldots, n$ takes two values: $\sigma(k)=0$ corresponds to the case when the segment $T_{k}$ is super-regular and $\sigma(k)=1$ corresponds to the case when the segment $T_{k}$ is not super-regular. On the sample space $\Omega$ we define two different probability spaces $\left(\Omega, \mathbf{P}_{1}\right)$ and $\left(\Omega, \mathbf{P}_{2}\right)$ by the following formulas:

$$
\mathbf{P}_{\mathbf{1}}\left(A^{j}\right)=\mathbf{P}_{\mathbf{1}}[\sigma(1), \ldots, \sigma(n)]=\mathbf{P}_{\mathbf{V}}[\sigma(1), \ldots, \sigma(n)]
$$

where $\mathbf{P}_{\mathbf{V}}$ is the Gibbs distribution $\mathbf{P}_{\mathbf{V}}$, corresponding to the boundary conditions $\varphi^{1}(x), x \in \mathbf{Z}^{\mathbf{1}}$, $\varphi^{\prime}(I), x \in I$ and

$$
\mathbf{P}_{\mathbf{2}}\left(A^{j}\right)=\mathbf{P}_{\mathbf{2}}[\sigma(1), \ldots, \sigma(n)]=q^{n-s}(1-q)^{s},
$$

where $s$ denotes the total number of 1 entries of the vector $A^{j}=[\sigma(1), \ldots, \sigma(n)]$.

We define a random vector $(\eta(1), \eta(2), \ldots, \eta(n))$ on the probability space $\left(\Omega, \mathbf{P}_{1}\right)$ and, respectively, a random vector $(\xi(1), \xi(2), \ldots, \xi(n))$ on the probability space $\left(\Omega, \mathbf{P}_{\mathbf{2}}\right)$ by the formulas:

$$
\eta(k)\left(A^{j}\right)=\sigma(k) \quad \text { and } \xi(k)\left(A^{j}\right)=\sigma(k) .
$$

The random variables $\eta(k)$ and $\xi(k)$ are defined on the same sample space but on different probability spaces.

Due to the definitions, the random variables $\eta(k)$ are dependent, and the random variables $\xi(k)$ are independent and identically distributed.

Consider the two sums $\sum_{k=1}^{n} \eta(k)$ and $\sum_{k=1}^{n} \xi(k)$.

Suppose that

$$
\mathbf{P}\left(\eta(m)=1 \mid \text { any conditions outside } T_{m}\right) \leqslant 1-q \text {. }
$$

Note that $\mathbf{P}\left(\eta(m)=1 \mid\right.$ any conditions outside $\left.T_{m}\right) \leqslant 1-q=\mathbf{P}(\xi(m)=1)$ and therefore the following natural lemma holds.

Lemma 11:

$$
\mathbf{P}\left(\sum_{k \in K} \eta(k) \geqslant l\right) \leqslant \mathbf{P}\left(\sum_{k \in K} \xi(k) \geqslant l\right)
$$

for all natural values of $l$.

The proof of the probabilistically clear Lemma is omitted. For the detailed proof see the Proposition in Ref. 15.

The random variables $\xi(k)$ are independent and identically distributed. The mathematical expectation of $\xi(k)$ equals $1-q$.

Now we show that

$$
P^{\mathrm{abs}}\left(\eta(m)=1 \mid \text { any conditions outside } T_{m}\right) \leqslant 1-q \text {. }
$$


Let $\mathbf{P}_{\mathbf{V}}$ be a Gibbs measure corresponding to arbitrary boundary conditions and $T_{k}$ be an arbitrary segment. Consider the set of all configurations on the interval $T_{k}$ and the restriction of the measure $\mathbf{P}_{\mathbf{V}}$ on this set. We show that at some value of $l$ the "absolute probability" $P^{\text {abs }}$ that in $T_{k}$ there is at least one regular segment $I_{k}$ is greater than $q>0$ for some constant $q$ not depending on $k$. The event $\eta(k)=1$ means that all segments belonging to $T_{k}$ are nonregular.

Suppose that a fixed configuration $\varphi^{\prime}\left(T_{m}\right)$ does not coincide with the ground state at all $I_{i}$ $\in T_{m}$.

The Peierls argument method directly imply that for some positive constant $t_{0}$

$$
P^{\mathrm{abs}}\left(\varphi^{\prime}\left(T_{m}\right) \mid \text { conditions outside } T_{m} \text { are } \varphi^{g r}(x)\right) \leqslant \exp \left(-\beta t_{0} l\right) .
$$

Note that when we increase the value of $l$ the influence of the conditions outside $T_{m}$ on the configuration in $T_{m}$ increases with the rate less than $l$ and therefore at some value of $l$ and for some positive constant $t$ we have

$$
P^{\text {abs }}\left(\varphi^{\prime}\left(T_{k}\right) \mid \text { any conditions outside } T_{m}\right) \leqslant \exp (-\beta t l) \leqslant 1-q_{0} .
$$

Thus, the probability $P^{\mathrm{abs}}\left(\eta(m)=1 \mid\right.$ any conditions outside $\left.T_{m}\right)$ as a union of at most $2^{l p}$ events with probabilities less than $1-q_{0}$, is bounded by some number $1-q$. The inequality (24) is proved.

Now Lemma 9 is a direct consequence of the strong law of large numbers for $\xi(k)$ and the Lemma 11. Indeed, consider independent Bernoulli trials when the probability of success at each trial is $1-q$. According to the law of large numbers, the probability of the event that the density of successes exceeds $1-q^{\prime} ; 0<q^{\prime}<q$, is less than $1 / 4$, when $V$ tends to infinity. It means that the "absolute probability" of the event that the density of non-super-regular segments $T_{k}$ is greater than $1-q^{\prime}$ is less than $1 / 4$. Due to Lemma 11 , this probability is greater than the $P^{\text {abs }}$ probability of the event that the density of non-super-regular segments $T_{m}$ is greater than $1-q^{\prime}$. In other words, the $P^{\text {abs }}$ probability of the event that the density of super-regular segments $T_{m}$ is less than $1-q^{\prime}$ is less than $1 / 4$. Thus, the $P^{\text {abs }}$ probability of the event that the density of super-regular segments $T_{m}$ is greater than $1-q^{\prime}$ is greater than $1 / 4$. Taking into account that each super-regular segment $T_{m}$ contains at least one regular segment, one can see that the last statement implies the Lemma 9 if the parameter $p$ is chosen from the open interval $\left(1-q^{\prime} / l, 1\right)$. We choose the value of $p$ as $1-q / 2 l$.

Lemma 9 is proved.

Proof of Lemma 10: Let us consider the set of all long clusters $D_{i}$ with the density of supports less than $p$. Let $\operatorname{supp}(D)=\cup_{i=j}^{r} \operatorname{supp}\left(K_{j}\right)$. These supports $K_{i}$ are connected between themselves and with the boundary. Since the density of supports is not greater than $p<1$, the sum of the lengths of bonds in both halves $[-V,-|I| / 2$ and $[|I| / 2, V]$ is not less than $(V-|I| / 2)(1-p)$. When $V$ goes to infinity the sum of lengths of bonds of any long cluster with the density less than $p$ tends to infinity. As it becomes apparent from the proof of Lemma $8 P^{\text {abs }}$ (Long, $>p$ ) does not exceed one. And it does not exceed one, if we omit the factor $g(x, y)$ corresponding to the long bond and since $g(x, y, \varphi)=\exp (-\beta f(x, y, \varphi))-1$ [see (12)] the impact of these bonds tends to zero. By choosing the appropriate value of $V$ we complete the proof of Lemma 10.

Lemma 10 is proved.

We omit the huge proof of Lemma 5 since it is absolutely analogous to the proof of Lemma 6. The only difference is the fact that in $\Xi^{1,{ }^{\prime}, 2,{ }^{\prime \prime}}$ overlapped clusters are allowed, so the density of nonregular segments of typical configurations in Lemmas 8,9 instead of $p$ will be a number less than $1-(1-p)(1-p)$.

Partition functions including only non-long-super clusters satisfy the following key lemma which has a geometrically-combinatorial explanation.

Lemma 12:

$$
\Xi^{1{ }^{\prime}, 2,,^{\prime \prime},(n . l .)}=Q \Xi^{1, ", 2,{ }^{\prime},(n . l .)}
$$


where the factor $Q=Q\left(\varphi^{1}(x), \varphi^{2}(x), \varphi^{\prime}(x), \varphi^{\prime \prime}(x)\right)$ is uniformly bounded: $0<$ const $_{1}<Q$ $<$ const $_{2}$.

The factor appears due to the fact that configurations with minimal energy corresponding to the different boundary conditions do not coincide everywhere (they coincide to within shifts, everywhere but finite area).

Proof of Lemma 12: Due to the constant $Q$ without loss of generality we assume that the configurations with minimal energy $\varphi_{\min }$ for both boundary conditions coincide.

According to the definitions and Lemma 4

$$
\Xi^{1,1^{\prime \prime},(n . l .)}=Q^{\prime} \sum^{*} w\left(S D_{1}\right) \cdots w\left(S D_{m}\right),
$$

where the summation is taken over all nonlong, nonordered compatible collections of super clusters.

According to the definition of the super cluster

$$
Q^{\prime} \sum^{*} w\left(S D_{1}\right) \cdots w\left(S D_{m}\right)=Q^{\prime} \sum^{1,{ }^{\prime}, *} w\left(D_{1}\right) \cdots w\left(D_{k}\right) \sum^{2,,^{\prime \prime} *} w\left(D_{1}\right) \cdots w\left(D_{l}\right)
$$

in $\Sigma^{1,{ }^{\prime}, *}$ and $\Sigma^{2,,^{\prime \prime}, *}$ the summation is taken over all nonordered collections of clusters $w\left(D_{1}^{1,{ }^{\prime}}\right) \cdots w\left(D_{k}^{1,{ }^{\prime}}\right)$ and $w\left(D_{1}^{2, \prime \prime}\right) \cdots w\left(D_{l}^{2, "}\right)$ such that their product belongs to $\Sigma^{*}$.

Similarly,

$$
\begin{aligned}
\Xi^{1, ", 2,{ }^{\prime},(n . l .)} & =Q^{\prime \prime} \sum^{* *} w\left(S D_{1}\right) \cdots w\left(S D_{m}\right) \\
& =Q^{\prime \prime} \sum^{1, ", * *} w\left(D_{1}\right) \cdots w\left(D_{k}\right) \sum^{2,{ }^{\prime}, * *} w\left(D_{1}\right) \cdots w\left(D_{l}\right) .
\end{aligned}
$$

In order to prove Lemma 12 we put one-to-one correspondence between $\Sigma * w\left(S D_{1}\right) \ldots w\left(S D_{m}\right)$ and $\Sigma * * w\left(S D_{1}\right) \ldots w\left(S D_{m}\right)$.
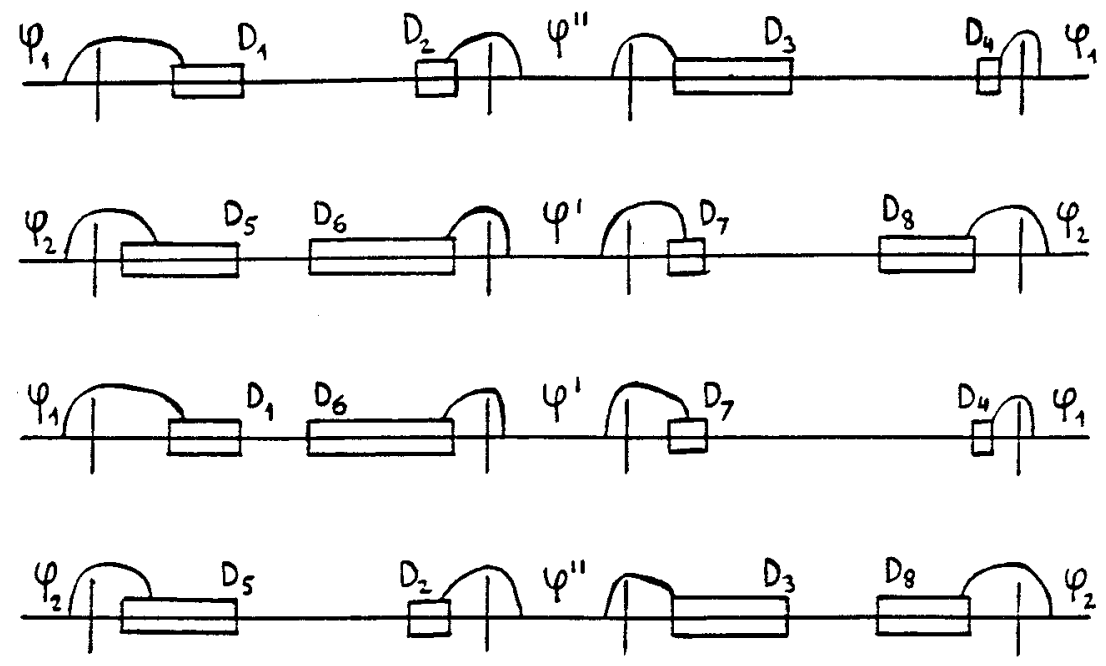

FIG. 1. 
Let us consider an arbitrary term $U=w\left(S D_{1}\right) \ldots w\left(S D_{a}\right)$ of $\Sigma^{*}$. By definitions

$$
U=w\left(D_{1}^{1,{ }^{\prime}}\right) \cdots w\left(D_{m}^{1, '}\right) w\left(D_{1}^{2, \prime \prime}\right) \cdots w\left(D_{k}^{2, "}\right),
$$

where the factor $w\left(D_{1}^{1,}\right) \cdots w\left(D_{m}^{1, \prime}\right)$ belongs to the $\Sigma^{1,{ }^{\prime}, *}$ and the factor $w\left(D_{1}^{2, " \prime}\right) \cdots w\left(D_{k}^{2,{ }^{\prime \prime}}\right)$ belongs to the $\Sigma^{2, ", *}$.

A cluster $D=\left[\left(K_{i}, i=1, \ldots, r\right) ; G^{\prime \prime}\right]$ is said to be basic, if the set $\left.\left(\left(\cup_{i=1}^{m} \operatorname{supp} D_{i}\right) \cup G^{\prime \prime}\right) \cap\left(\left(\mathbf{Z}^{1}-I_{N}\right) \cup I\right)\right)$ is not empty. In Fig. 1 all clusters are basic.

Consider the set of all clusters $\mathbf{W}(U)$ of the term $U: \mathbf{W}(U)=\cup_{i=1}^{m} D_{i}^{1, "} \cup_{i=1}^{k} D_{i}^{2,}$ and four subsets of $\mathbf{W}(U)$ :

$$
\begin{gathered}
W^{\prime}=\left[D^{\prime}=\left[\left(K_{i}, i=1, \ldots, r\right) ; G^{\prime}\right] \in \Xi^{2,,^{\prime}}:\left(\left(\bigcup_{i=1}^{k} \operatorname{supp} D_{i}\right) \cup G^{\prime}\right) \cap I \text { is not empty }\right], \\
W^{\prime \prime}=\left[D^{\prime \prime}=\left[\left(K_{i}, i=1, \ldots, r\right) ; G^{\prime}\right] \in \Xi^{11^{\prime \prime}}:\left(\left(\bigcup_{i=1}^{m} \operatorname{supp} D_{i}\right) \cup G^{\prime}\right) \cap I \text { is not empty }\right], \\
W^{1}=\left[D^{1}=\left[\left(K_{i}, i=1, \ldots, r\right) ; G^{\prime}\right] \in \Xi^{11^{\prime \prime}}:\left(\left(\bigcup_{i=1}^{m} \operatorname{supp} D_{i}\right) \cup G^{\prime}\right) \cap\left(\mathbf{Z}^{1}-I_{N}\right) \text { is not empty }\right], \\
W^{2}=\left[D^{2}=\left[\left(K_{i}, i=1, \ldots, r\right) ; G^{\prime}\right] \in \Xi^{2,,^{\prime}}:\left(\left(\bigcup_{i=1}^{k} \operatorname{supp} D_{i}\right) \cup G^{\prime}\right) \cap\left(\mathbf{Z}^{1}-I_{N}\right) \text { is not empty }\right] .
\end{gathered}
$$

Note that the subsets $W^{\prime}, W^{\prime \prime}, W^{1}, W^{2}$ contain only basic clusters and the union of them contain all basic clusters of the term $U$.

Let us consider an arbitrary term $U=w\left(S D_{1}\right) \cdots w\left(S D_{b}\right)$ of $\Sigma^{* *}$. By the definitions

$$
U^{\prime}=w\left(D_{1}^{1, "}\right) \cdots w\left(D_{l}^{1,{ }^{\prime \prime}}\right) w\left(D_{1}^{2,{ }^{\prime}}\right) \cdots w\left(D_{n}^{2,{ }^{\prime}}\right),
$$

where the factor $w\left(D_{1}^{1, "}\right) \cdots w\left(D_{m}^{1, \prime \prime}\right)$ belongs to the $\Sigma^{1,{ }^{\prime \prime}, * *}$ and the factor $w\left(D_{1}^{2,}\right) \cdots w\left(D_{k}^{2,{ }^{\prime}}\right)$ belongs to the $\Sigma^{2,{ }^{\prime}, * *}$.

Consider the set of all clusters $W\left(U^{\prime}\right)$ of the term $U^{\prime}: W\left(U^{\prime}\right)=\cup_{i=1}^{m} D_{i}^{1, "} \cup_{i=1}^{k} D_{i}^{2,}$. In just the same way we can define four subsets of $W\left(U^{\prime}\right)$.

Consider a term $U=w\left(D_{1}\right) \cdots w\left(D_{k}\right) \in \Sigma^{*}$, containing only basic clusters. By definition $\cup_{i=1}^{k} D_{i}$ can be represented as $\cup_{i=1}^{k} D_{i}=\left(\cup_{i=1}^{m} D_{i}\right) \cup\left(\cup_{i=m+1}^{k} D_{j}\right)$, where the clusters $\cup_{i=1}^{m} D_{i}$ $=W^{1} \cup W^{\prime}$; and $\cup_{i=m+1}^{k} D_{j}=W^{2} \cup W^{\prime \prime}$.

From the definition of nonlong clusters and $W^{\prime}, W^{\prime \prime}, W^{1}, W^{2}$ it easily follows that there exists the same term $U^{\prime}=w\left(D_{1}\right) \ldots w\left(D_{k}\right) \in \Sigma^{* *}$, such that $\cup_{i=1}^{k} D_{i}=\left(\cup_{i=1}^{m} D_{i}\right) \cup\left(\cup_{i=m+1}^{k} D_{j}\right)$, where the clusters $\cup_{i=1}^{m} D_{i}=W^{1} \cup W^{\prime}$; and $\cup_{i=m+1}^{k} D_{j}=W^{2} \cup W^{\prime \prime}$.

Figure 1 shows four collections of clusters $\mathrm{COL}_{1}=\left[D_{1}^{1, "}, D_{2}^{1,{ }^{\prime \prime}}, D_{3}^{1, " \prime}, D_{4}^{1, "}\right], \mathrm{COL}_{2}$ $=\left[D_{5}^{2,{ }^{\prime}}, D_{6}^{2,{ }^{\prime}}, D_{7}^{2,{ }^{\prime}}, D_{8}^{2, \prime}\right], \mathrm{COL}_{3}=\left[D_{1}^{1,{ }^{\prime}}, D_{6}^{1,{ }^{\prime}}, D_{7}^{1,{ }^{\prime}}, D_{4}^{1,{ }^{\prime}}\right], \mathrm{COL}_{4}=\left[D_{5}^{2, \prime \prime}, D_{2}^{2, \prime \prime}, D_{3}^{2,{ }^{\prime \prime}}, D_{8}^{2, \prime \prime}\right]$.

Two coincident terms $U=U^{\prime}=\Pi_{i=1}^{8} w\left(D_{i}\right)$ belonging to the sums $\Sigma^{*}$ and $\Sigma^{* *}$ are constructed by the Cartesian product of the collections $\mathrm{COL}_{1}, \mathrm{COL}_{2}$, and $\mathrm{COL}_{3}, \mathrm{COL}_{4}$, respectively.

We see that between terms $U \in \Sigma^{*}$ and $U^{\prime} \in \Sigma^{* *}$ containing only basic clusters we easily can put a one-to-one correspondence.

Consider a term $U=w\left(D_{1}\right) \cdots w\left(D_{k}\right) w\left(D_{k+1}\right) \cdots w\left(D_{n}\right) \in \Sigma^{*}$, containing basic clusters $D_{1} \cdots D_{k}$ and not basic clusters $D_{k+1} \cdots D_{n}$.

It can be easily shown that there exists a term $U^{\prime}=w\left(D_{1}\right) \cdots w\left(D_{k}\right) w\left(D_{k+1}\right) \cdots w\left(D_{n}\right)$ $\in \Sigma^{* *}$ coinciding with the term $U \in \Sigma^{*}$. Indeed, suppose that there is no term $U^{\prime}$ 
$=w\left(D_{1}\right) \cdots w\left(D_{k}\right) w\left(D_{k+1}\right) \cdots w\left(D_{n}\right) \in \Sigma^{* *}$ coinciding with the term $U \in \Sigma^{*}$. Then, according to the definition of the long clusters, we directly get that, the term $U$ contains long super cluster, which contradicts the definition of $\Sigma^{*}$.

Lemma 12 is proved.

Remark: The essential point of the proof of the important Lemma 12 (therefore, of this paper) is the amusing fact that $\Sigma * w\left(S D_{1}\right) \cdots w\left(S D_{m}\right)$ and $\Sigma * * w\left(S D_{1}\right) \cdots w\left(S D_{m}\right)$ coincide.

Now the demanded inequality (18) is a direct consequence of Lemmas 5 and 12 . The inequality (18), therefore Lemma 3 is proved.

Let $\mathbf{P}^{1}$ and $\mathbf{P}^{\mathbf{2}}$ be two different extreme Gibbs states of the model (1) corresponding to the boundary conditions $\varphi^{1}(x)$ and $\varphi^{2}(x)$, respectively.

Theorem 5: (Ref. 16.) $\mathbf{P}^{1}$ and $\mathbf{P}^{\mathbf{2}}$ are singular or coincide.

Proof of Theorem 4: Let $\mathbf{P}^{\mathbf{1}}$ and $\mathbf{P}^{\mathbf{2}}$ be two different extreme Gibbs states of the model (1) corresponding to the boundary conditions $\varphi^{1}(x)$ and $\varphi^{2}(x)$ respectively. According to Lemma 3 $\mathbf{P}^{1}$ and $\mathbf{P}^{2}$ are not singular. Therefore, according to Theorem $5 \mathbf{P}^{\mathbf{1}}$ and $\mathbf{P}^{\mathbf{2}}$ coincide, which contradicts the assumption. Theorem 4 is proved.

\section{UNIQUENESS OF GIBBS STATES}

In this section we prove the main Theorem 1.

The statement of Theorem 1 for rational densities coincides with Theorem 4. Thus, in order to complete the proof of Theorem 1, we have to prove the following theorem, which covers the case when the density of the special ground state is irrational.

Theorem 6: Suppose that the value of the external field $\mu$ of the model (1) belongs to the set $\mathbf{C}^{\mathrm{ir}}=\mathbf{R}^{1}-\cup_{\kappa}\left(\mu_{\kappa}^{-}, \mu_{\kappa}^{+}\right)$. Then the model (1) has a unique Gibbs state at all values of the temperature $\beta^{-1}$.

It can be easily shown that the special ground states of the model (1) are not stable when the density is irrational. In other words, the Peierls constant $t$ for the special ground state tends to zero, when $p \rightarrow \infty$. The essence of this fact is the following.

For the fixed irrational number $\eta=\left[n_{0}, n_{1}, \ldots, n_{s}, \ldots\right]$ consider the corresponding special ground state $\varphi_{\kappa}(x)$ and its arbitrary perturbation $\varphi_{\kappa}^{\prime}(x)$. The configuration $\varphi_{\kappa}^{\prime}(x)$ is not a special ground state, therefore for some pair of points, say $x$ and $y \in \mathbf{Z}^{1} ; \varphi_{\kappa}^{\prime}(x)=\varphi_{\kappa}^{\prime}(y)=1$, we have a violation of Hubbard's criterion. Let $x$ and $y$ be closest points with this property. When the distance between $x$ and $y$ tends to infinity, the Peierls constant tends to zero.

In the irrational case the special ground states are not stable, but this fact is not crucial for our method. Since the essence of our method is the estimation of long super clusters connecting the boundary with the segment $I$, small clusters not satisfying Peierls condition cannot "help" to connect the boundary with $I$, and it turns out that big clusters satisfy the Peierls stability condition and the method works. One can say that the special ground states in the irrational case are "stable in general."

Below we give the mathematical details of the last observation.

Consider $\eta(s)=\left[n_{0}, n_{1}, \ldots, n_{s}\right]$.

Lemma 13: Suppose that the value of the external field $\mu$ of the model (1) belongs to the interval $\left(\mu_{\kappa(s)}^{-}, \mu_{\kappa(s)}^{+}\right)$for some number $\kappa(s)=\eta(s)^{-1}$. Let $\varphi^{\prime}(x)$ be an arbitrary finite perturbation of the special ground state $\varphi_{\kappa(s)}(x)$ such that the boundary $B$ of the configuration $\varphi^{\prime}(x)$ includes a unique contour $K$. Then there exists a positive constant $t_{s}$ depending only on the Hamiltonian (1), such that

$$
H\left(\varphi^{\prime}(x)\right)-H\left(\varphi_{\kappa(s)}(x)\right) \geqslant t_{s}|\operatorname{supp} B|
$$

where $|\operatorname{supp} B|$ is the total area of the support of the boundary.

Lemma 13 was proved in Ref. 13 [see Lemma 1 and Sec. 5 (Ref. 13)].

Thus, for each nonnegative integer $s$ the number $t_{s}$ is defined. Suppose that a positive number $t$ less than $t_{1}$ is fixed. Let $s$ be the maximal number meeting the condition $t_{s}>t$.

Now we are ready to define the notion of a contour in the irrational case. 
Let us consider an arbitrary configuration $\varphi(x)$. Let $C=\cup_{i \in \operatorname{Ind}}\left[x_{i}, y_{i}\right]$, where $x_{i}, y_{i} \in \mathbf{Z}^{1}$ and $x_{i} \neq y_{i}$ has the following properties:

(1) For each segment $\left[a_{i}, b_{i}\right]$ from the set $\mathbf{Z}^{\mathbf{1}}-C$ there exists a special ground state $\varphi_{\kappa}$, such that the restriction of this configuration on $\left[a_{i}, b_{i}\right]$ coincides with $\varphi\left(\left[a_{i}, b_{i}\right]\right)$.

(2) For any $C^{\prime} \subset C ; C^{\prime} \neq C$ the property 1 is not held.

It can be easily shown that the set $C=C(\varphi(x))$ is not uniquely defined. Suppose that, some rule uniquely determines the set $C$ for each configuration $\varphi(x)$. Let $\mathbf{Z}^{1}-C=\cup_{i}\left[a_{i}, b_{i}\right]$. We say that $\varphi\left(\left[a_{i}, b_{i}\right]\right)$; is a preregular phase. Consider any segment $\left[x_{i}, y_{i}\right]$ belonging to $C$. The segment $\left[x_{i}, y_{i}\right]$ is said to be $t$-negligible, if for each segment $\left[\mathrm{v}_{i}, w_{i}\right]$ covering $\left[x_{i}, y_{i}\right], w_{i}-\mathrm{v}_{i}=p[p$ is the numerator of $\eta(s)]$ there exists a special ground state $\varphi_{\kappa(s)}$, such that the restriction of this configuration on $\left[\mathrm{V}_{i}, w_{i}\right]$ coincides with $\varphi\left(\left[\mathrm{V}_{i}, w_{i}\right]\right)$. Let $C=\cup_{i \in \operatorname{Ind}}\left[x_{i}, y_{i}\right]=\left(\cup_{i \in \operatorname{Ind}(t)}\right.$ $\left.\times\left[x_{i}, y_{i}\right]\right) \cup\left(\cup_{i \in \operatorname{Ind}-\operatorname{Ind}(t)}\left[x_{i}, y_{i}\right]\right)$, where $\operatorname{Ind}(t)$ means that the union is taken over all $t$-negligible segments. The support of the preboundary supp $P B$ of the configuration $\varphi(x)$ will be defined as $\operatorname{supp} P B=\left(\cup_{i \in \operatorname{Ind}(t)}\left[x_{i}, y_{i}\right]\right) \cup\left(\cup_{i \in \operatorname{Ind}-\operatorname{Ind}(t)}\left[x_{i}-d_{0} p, y_{i}+d_{0} p\right]\right)=\operatorname{supp} P B($ main $) \cup \operatorname{supp} P B(t)$. Each segment belonging to the union $\operatorname{supp} P B$ will be called a support of a precontour and is denoted by $\operatorname{supp} P K$. The support $\left[x_{i}, y_{i}\right]$ of a precontour is said to be $t$-negligible, if $\left[x_{i}, y_{i}\right]$ belongs to $\operatorname{supp} P B(t)$.

We define contours as in the Definition 1 . The constants $p, d_{0}$ and $N_{b}$ for irrational density $\eta^{-1}$ will be constants defined for rational density $\eta(s)^{-1}$.

The pair $P K=\left(\operatorname{supp} P K, \varphi^{\prime}(\operatorname{supp} P K)\right)$ is called a precontour. The set of all precontours is called a preboundary $P B$ of the configuration $\varphi^{\prime}(x)$. Two precontours $P K_{1}$ and $P K_{2}$ are said to be connected if $\operatorname{dist}\left(\operatorname{supp} P K_{1}, \operatorname{supp} P K_{2}\right)<N_{b}$ and at least one of them is not $t$-negligible. The set of precontours $\left(P K_{i} ; i \in \mathrm{Ind}\right)$ is called connected if for any two precontours $P K_{c}$ and $P K_{d} ; c, d$ $\in$ Ind there exists a collection $\left(P K_{j_{1}}=P K_{c}, \ldots, P K_{j_{i}}, \ldots, P K_{j_{n-1}}, P K_{j_{n}}=P K_{d}\right) ; j_{i} \in$ Ind, $i$ $=1, \ldots, n$; such that any two precontours $P K_{j_{i}}$ and $P K_{j_{i+1}}, i=1, \ldots, n-1$ are connected. Let $\cup_{i=1}^{n} P K_{i}$ be some maximal connected component of the preboundary $P B$. Suppose that $\operatorname{supp} P K_{i}=\left[a_{i}, b_{i}\right]$ and $b_{i}<a_{i+1} ; i=, \ldots, n-1$.

The pair $K=\left(\operatorname{supp} K, \varphi^{\prime}(\operatorname{supp} P K)\right)$, where supp $K=\left[a_{1}, b_{n}\right]$ is called a contour. The set of all contours is called a boundary $B$ of the configuration $\varphi^{\prime}(x)$.

A contour is said to be $t$-negligible, if its support is $t$-negligible.

By the definitions, the distance between the supports of two $t$-negligible contours exceeds $p$, where $p$ is the numerator of $\eta(s)$ and the length of the support of any $t$-negligible contour is one.

The following lemma is reformulation of Lemma 13 for irrational densities.

Lemma 14: Suppose that the value of the external field $\mu$ of the model (1) belongs to the set $\mathbf{C}^{\mathrm{ir}}=\mathbf{R}^{\mathbf{1}}-\cup_{\kappa}\left(\mu_{\kappa}^{-}, \mu_{\kappa}^{+}\right)$. Let $\varphi^{\prime}(x)$ be an arbitrary finite perturbation of the special ground state $\varphi_{\kappa}(x)$ such that the boundary $B$ of the configuration $\varphi^{\prime}(x)$ includes a unique contour (not $t$-negligible contour) $K$. Then there exists a positive constant $t_{s}$ depending only on the Hamiltonian (1), such that

$$
H\left(\varphi^{\prime}(x)\right)-H\left(\varphi_{\kappa}(x)\right) \geqslant t_{s}|\operatorname{supp} B|
$$

where $|\operatorname{supp} B|$ is the total area of the support of the boundary.

Suppose that the value of the external field $\mu$ of the model (1) belongs to the set $\mathbf{C}^{\mathrm{ir}}=\mathbf{R}^{1}$ $-\cup_{\kappa}\left(\mu_{\kappa}^{-}, \mu_{\kappa}^{+}\right)$. Let $t, 0<t<t_{1}$ is fixed and $t_{s}$ is chosen as above.

Lemma 15: Let $\varphi_{\min }(x) \in \Phi(V)$ be a configuration with the minimal energy:

$$
H\left(\varphi_{\min }(x) \mid \varphi^{1}(x)\right)=\min _{\varphi(x) \in \Phi(V)} H\left(\varphi(x) \mid \varphi^{1}(x)\right) .
$$

Then the configuration $\varphi_{\min }(x)$ has the following structure:

The restriction of the configuration $\varphi_{\min }(x)$ on the set $\left[-V+N_{b}, V-N_{b}\right]$ contains $t$-negligible contours and $p-1$ non t-negligible contours, moreover the sum of weights of all t-negligible 
contours is bounded by constant, not depending on the boundary conditions, all of $p-1$ non $t$-negligible contours are interface contours $I K_{i}, i=1, \ldots, m$, where $m<p-1$ and $\left|\operatorname{supp} I K_{i}\right|$ $<3 d_{0} p+N_{b}$.

The proof of Lemma 15 is very similar to the proof of Lemma $1^{13}$ and will be omitted.

From Lemma 15 follows that the density of possible $t$-negligible contours of $\varphi_{\min }(x)$ tends to zero, when $V$ goes to infinity.

Now the proof of Theorem 6 principally coincides with the proof of Theorem 3 and will be omitted. Theorem 6 , and hence main Theorem 1 is proved.

\section{FINAL REMARKS}

The unique limit Gibbs state of the model (1) is translationally invariant. This result was proved independently in Ref. 1 by using of the method of the equivalence of boundary conditions, ${ }^{17}$ and in Ref. 11 by using of energy-entropy inequalities.

At low temperatures, the sum of the statistical weights of all clusters having fixed support has an exponential estimation (see Lemma 16, Ref. 13) and each limit Gibbs state of the model (1) is a "small perturbation of special ground states" (see Lemma 17, Ref. 13).

The essential points in the proof of the uniqueness of Gibbs states are the geometrically combinatorial Lemma 12 and the estimation of long super clusters, connecting the boundary with the segment $I$. This estimation mainly works due to the fact that ground states of the model (1) degenerate. In Ref. 13 we proved Theorem 4 at low temperatures. The temperature restriction was related with the fact that at low temperatures the weight of the support of a cluster has an exponential estimation [Lemmas 16 and 17 (Ref. 13)] and hence long clusters are negligible (Ref. 13). But at any temperature an exponential estimation is absent. In the general case, when we estimate the statistical weight of long super clusters, a key role plays the Lemma 6 on the estimation of the value of the interaction between contours.

In Ref. 15 at low temperatures the result of Ref. 13 is extended to more abstract models. The method of the proof of Theorem 1 shows that the result of Ref. 15 can be extended to all values of the temperatures.

\footnotetext{
${ }^{1}$ S. E. Burkov and G. Ya. Sinai, Usp. Mat. Nauk 38, 205 (1983).

${ }^{2}$ R. L. Dobrushin, Teor. Veroyatn. Primen. 18, 201 (1968).

${ }^{3}$ R. L. Dobrushin, Funk. Anal. Pril. 2, 44 (1968).

${ }^{4}$ D. Ruelle, Commun. Math. Phys. 9, 267 (1968).

${ }^{5}$ F. Dyson, Commun. Math. Phys. 12, 91 (1969).

${ }^{6}$ F. Dyson, Commun. Math. Phys. 21, 269 (1971).

${ }^{7}$ J. Hubbard, Phys. Rev. B 17, 494 (1978).

${ }^{8}$ P. L. Pokrovsky and G. V. Uimin, J. Phys. C 11, 3535 (1978).

${ }^{9}$ P. Bak, "Incommensurate, commensurate and chaotic phases," preprint, Nordita, 1982.

${ }^{10} \mathrm{P}$. Bak and R. Bruinsma, Phys. Rev. Lett. 49, 249 (1982).

${ }^{11}$ M. Fannes, P. Vanheuverzwijn, and A. Verbeure, J. Stat. Phys. 29, 547 (1982).

${ }^{12}$ A. A. Kerimov, Teor. Mat. Fiz. 58, 473 (1984).

${ }^{13}$ A. A. Kerimov, J. Stat. Phys. 72, 571 (1993).

${ }^{14}$ J. Bricmont, K. Kuroda, and J. L. Lebowitz, Commun. Math. Phys. 101, 501 (1985).

${ }^{15}$ A. A. Kerimov, Physica A 258, 183 (1998).

${ }^{16}$ H.-O. Georgee, Gibbs Measures and Phase Transitions (de Gruyter, Berlin, New York, 1988).

${ }^{17}$ J. Bricmont, J. L. Lebowitz, and C. E. Pfister, J. Stat. Phys. 21, 573 (1979).
} 